\title{
Sec3 Exocyst Component Knockdown Inhibits Axonal Formation and Cortical Neuronal Migration During Brain Cortex Development
}

\section{Florentyna Bustos Plonka}

Department of Biological Chemistry Ranwel Caputto, School of Chemical Sciences, National University of Cordoba and CIQUIBIC-CONICET, X5000HUA, Ciudad Universitaria, Córdoba, Argentina.

\section{Lucas Sosa}

Department of Biological Chemistry Ranwel Caputto, School of Chemical Sciences, National University of Cordoba and CIQUIBIC-CONICET, X5000HUA, Ciudad Universitaria, Córdoba, Argentina.

\section{Santiago Quiroga ( $\square$ santiago.quiroga@unc.edu.ar)}

Department of Biological Chemistry Ranwel Caputto, School of Chemical Sciences, National University of Cordoba and CIQUIBIC-CONICET, X5000HUA, Ciudad Universitaria, Córdoba, Argentina.

\section{Research Article}

Keywords: exocyst, axonal, migration, brain

Posted Date: February 5th, 2021

DOI: https://doi.org/10.21203/rs.3.rs-149990/v1

License: (c) (1) This work is licensed under a Creative Commons Attribution 4.0 International License. Read Full License 
Sec3 exocyst component knockdown inhibits axonal formation and cortical neuronal migration during brain cortex development

\author{
Florentyna Bustos Plonka ${ }^{1}$, Lucas J. Sosa ${ }^{1 *}$ and Santiago Quiroga ${ }^{1 *}$ \\ 1 Department of Biological Chemistry Ranwel Caputto, School of Chemical Sciences, \\ National University of Cordoba and CIQUIBIC-CONICET, X5000HUA, Ciudad \\ Universitaria, Córdoba, Argentina.
}

*Send correspondence to: $\underline{\text { santiago.quiroga@ unc.edu.ar and/or lucas.sosa@ unc.edu.ar }}$

SQ ORCID: 0000-0001-9087-7591 


\section{Abstract}

During neuronal development cell polarization is of vital importance. The establishment of polarity necessitates initial axonal outgrowth in concomitance with the addition of new membrane to the axon's plasmalemma. Axolemmal expansion occurs by exocytosis of plasmalemmal precursor vesicles primarily at the neuronal growth cone membrane. The multiprotein exocyst complex drives spatial location and specificity of vesicle fusion at plasma membrane. However, the specific participation of its different proteins on neuronal differentiation has not been fully established. In the present work we analyzed the role of Sec3, a prominent exocyst complex protein on neuronal differentiation. Using mice hipocampal primary cultures we determined that $\operatorname{Sec} 3$ is expressed in neurons at early stages prior to neuronal polarization. Furthermore, we determined that silencing of Sec3 in mice hippocampal neurons in culture precluded polarization. Moreover, using in utero electroporation experiments, we determined that Sec3 knockdown affected cortical neurons migration and morphology during neocortex formation. Our results demonstrate that the exocyst complex protein Sec3 plays an important role in axon formation in neuronal differentiation and the migration of neuronal progenitors during cortex development.

\section{Introduction}

The formation of a polarized neuron, containing a single long axon and several branching dendrites, requires the action of two interrelated processes, specification of the axon and initial axon outgrowth and the addition of new membrane to the axon's plasmalemma. Axolemmal expansion occurs by exocytosis of plasmalemmal precursor 
vesicles (PPVs) primarily at the neuronal growth cone [1-3], a process regulated by IGF1 activation of the phosphatidylinositol-3 kinase pathway [4-6]. Exocytosis requires two vital processes for proper cell function, vesicle attachment and vesicle fusion [7, 8]. Before vesicle attachment, a physical link between the vesicle and the membrane is required in order to bring them closer and facilitate (directly or indirect) SNARE mediated fusion $[9,10]$. Diverse studies in yeast and mammals have determined that the exocyst plays a key role in the polarized membrane traffic. It is well established that a tethering complex, the exocyst complex (EC), drives spatial location and specificity of vesicle fusion at the plasma membrane where the PPVs are directed [11-15]. The exocyst is a well conserved octameric complex usually comprised by Sec3, Sec5, Sec6, Sec8, Sec10, Sec15, Exo70 and Exo84. While several studies have been made to determine the structure of the complex as well as the assembly process $[9,16-19]$, it still remain unclear the role of the EC in one of the most polarized cell; the neuron.

We have previously reported that, in cultured hippocampal neurons, a universally employed method to study neuronal development and polarization, TC10 activation by IGF - 1 and the consequent assembling of Exo70 to the growth cone plasmalemma are critical for the control of PPVs exocytosis and, hence, pioneer axonal outgrowth and the establishment of neuronal polarity [20]. It has been also shown by other colleagues that, exocyst proteins, such as Sec6 and Sec8, are concentrated at growth cones of hipocampal neurons [14, 21, 22]. Moreover, in PC12 cells, proteins of the EC targets secretory vesicles to specific domains of the plasma membrane through its association with the microtubules, promoting neurite outgrowth [21]. Also, a NGF - induced Exo70 - TC10 complex has been shown to modulate neurite spin-off in PC12 cells [23]. In cortical neurons, cells expressing undetectable levels Sec6, Sec8 and Exo84 failed to develop a Tau1 positive axon [24]. Additionally, the EC (especially the Sec6, Sec8 and Exo84 
proteins), interact with the polarity complex (Par3 - Par6), a major controller of neuronal polarization $[24,25]$. Altogether, these results imply that the exocyst complex is critical for neurite development and neuronal polarization [26].

However, the identification in neurons of the key components of the exocyst complex that could participate in the regulation of initial axonal outgrowth and the establishment of neuronal polarity has not yet been fully achieved. One of the key elements of the exocyst complex is the Sec3 protein which is necessary for the dynamic formation of one of the two subcomplexes that conform the exocyst complex in order to tethering the vesicles to the membrane $[27,28]$.

In this context, the potential role of $\sec 3$ this protein in neuronal differentiation has not been yet studied. Here, we examined the expression and distribution of Sec3 in hippocampal neurons in culture and its participation in neuronal differentiation and polarity. Exocyst complex protein was expressed early in developing neurons and present in the growth cone and distal axon, prominent sites for new membrane addition. In these cells, loss of function of Sec3 repressed the establishment of neuronal polarity. Using in utero electroporation we also studied the consequences of Sec3 loss of function on pyramidal cortex neuron migration and changes in polarity during cortical formation. Neurons electroporated with a shRNA targeting Sec3 failed to migrate to the upper cortical layers and accumulated mainly at the ventricular/subventricular zones. Knocking down Sec3 also abrogated the morphological change from multipolar to bipolar and cells were arrested as multipolar forming atypical tissue organization. In summary, the results reported in this study show that Sec3 is necessary for axonal outgrowth and the establishment of neuronal polarity in hippocampal neurons in culture and the early polarity switch of cortical plate neurons during cortical formation in utero. 


\section{Results}

\section{Expression and distribution of Sec3 in developing neurons}

In order to be involved in neuronal polarization, a protein should be expressed early before this phenomenon occurs, so we assessed wheatear Sec3 protein is expressed in cultured hippocampal pyramidal neurons at early stages of development. In this model, cells undergo stereotypical changes in the morphology from symmetrical or not polarized neurites (between 12 and 18 hours of differentiation in vitro (H.I.V)) to exhibit a discernible axon (20-24 h in culture); and polarized morphology [29]. First we analyzed the expression of Sec3 at 18 or 30 H.I.V (stage 2 and stage 3 respectively) by immunofluorescence experiments. Immunostaining with $\mathrm{Sec} 3$ antibodies showed that there is a relatively prominent expression of this protein at 18 H.I.V (Figure 1 a -top). After 30 H.I.V neurons are in stage 3 exhibiting relatively long axons and Sec3 is expressed in the cells, especially in axons (Fig. 1 a-bottom). Also we determined by Western Blot analysis of cell lysates harvested at different times of culture that Sec3 is expressed previous to the polarization event, during the polarization ( 24 and $36 \mathrm{~h}$ ) and after the polarization; presenting a significant diminution at $72 \mathrm{~h}$ when the cells are fully polarized (Fig. 1 b and 1c).

Previously published data indicated that Sec6, Sec8 and Exo70 are localized in the axon and axon growth cones of hipocampal neurons $[14,20,21]$. The immunofluorescence showed in Fig.1a shows the enrichment and the preferentially distribution of Sec3 in the axon and the growth cone, a neuronal domain that leads the axonal growth and prominent site for the addition of new membrane in developing neurons $[14,20$, 21]. In this context more evidence about the expression of Sec 3 at the axon was obtained by fluorescent microscopy and imaging colocalization analysis with Tau1, enriched at the distal 
axons. To determine wheatear Sec3 co-distribute with Tau1, stage 3 neurons were stained with Sec3 (green channel) and Tau1 antibody (red channel) (Figure 2 a-top). As we can observe, a strong colocalization between these two proteins. (Figure 2 a -bottom) were found in the neuronal axon (Figure 2b- left). The histogram reveals a high spatial concordance between the two antibody marks (Figure $\mathbf{2 b}$ - right). The quantitative colocalization analysis was conducted to determined Pearson's (PC), Mander's (MC) and Fractionary Manders (FM) colocalization parameters (Fig. 2 c). Statistical analysis data demonstrated that all coefficients are in concordance of a positive colocalization between Sec3 and Tau1. We then evaluated the expression and subcellular distribution of Sec3 in the growth cone. The immunofluorescence performed at late stage $2 /$ early stage 3 (when the growth cone is more prominent) using Sec3 and Tubulin antibody showed a robust expression of the former at the growth cone (Fig. $\mathbf{2}$ d). The presence of this protein at the growth cone was also assessed by subcellular fractioning of fetal brain, especially in the axonal growth cone fraction (GCPs) [30,31]. Our results showed that Sec3 is present in the GCPs (Fig. 2 e). These results further confirmed that Sec3 has a preferentially distribution at the axon and is present at the growth cone.

\section{Sec3 is necessary for the in the establishment of neuronal polarity}

Further investigation regarding the possible involvement of $\operatorname{Sec} 3$ on the establishment of neuronal polarity was conducted. We silenced the expression of Sec3 protein in mice hippocampal neuron cultures using targeted shRNAs inserted into bicistronic plasmid which has a second promoter within the same vector that expressed enhanced green fluorescent protein (GFP). When the polarity is impaired, the neurons remain mostly at early stages and it can be assessed by the lack of one long prolongation (at least 20um larger than the others prolongations) and the absence of Tau1 protein enriched at one particular neurite. Transfection of Neuro-2a cells with Sec3-targeted shRNA significantly decreased Sec3 
protein in cultures (Fig 3c). The transfected neurons expressed virtually no detectable Sec3 and failed to form axons; only short, minor neurites were present. Note also the lack of enrichment of Tau-1 in any particular neurite of the transfected neurons (Fig. 3 a toparrow). In contrast, neurons transfected with a scrambled RNA sequence (ssCtrl) inserted in the same plasmid generated a long Tau-1 positive process (Fig.3a bottom). Cotransfection of neurons with shSec3 and Sec3FL (a cDNA enconding for the synthesis of human Sec3) rescued the morphology with the outgrowth of an axon enriched in Tau-1 (Fig3b). To analyze these observations quantitatively we classified the neurons into different stages of development and compared the neurons transfected with Sec3-targeted shRNAs to neurons in the same cultures containing control shRNA or co-transfected with Sec3FL and shSec3 after 36 H.I.V. We found that over $80 \%$ of the Sec3-targeted shRNAs transfected neurons remained at stages 1 or 2 of differentiation, and less than $20 \%$ had formed a discernible axon. In contrast, over $50 \%$ of the control exhibited a relatively long Tau-1containing axon. Regarding the cells cotransfected with ahSec3 and Sec3FL about $40 \%$ of the cells exhibited a discernible Tau-1 enriched axons (Fig. 3 d).

One of the earliest events in the differentiation of hippocampal neurons is-is the enrichment of phosphorylated (active) insulin like growth factor type 1 receptor (IGF-1r) in a single minor neurite. Upon IGF1r phosphorylation, cells do not exhibit a noticeable axon (Stage 2 of development) nonetheless the future axon is discernible since the active receptor is specifically segregated to one neurite [32]. Hence, we next analyzed the distribution of active IGF-1r in control neurons or in neurons transfected with shRNA directed to Sec3. As anticipated, the polarized distribution of the active IGF-1r was evident in the neurons transfected with a non-relevant shRNA control sequence (ssCtrl) (Fig. 3e. bottom-arrow). In the neurons transfected with Sec3 targeted shRNA (Fig. 3e.top labeling of the active IGF1r was not enriched in any particular minor process. In both experiments cells were deprived 
of growth factors for $4 \mathrm{~h}$ and challenged for 2 min with 20nM IGF-1. The active IGF-1r polarization index (for a description see Fig. 3 legend) was significantly higher $(\mathrm{p} \leq 0.001)$ in the neurons transfected with the ssCtrl sequence than in the Sec3 suppressed neurons (Fig. 3f).

Taken together, these results indicate that $\operatorname{Sec} 3$ is essential for axonal growth and the establishment of polarity in hippocampal neurons.

\section{Sec3 is necessary for the regulation of cortical neuron migration}

Changes in polarity and morphology have been largely analyzed in cells in culture, so we wanted to verify our findings in an in situ model. The most studied in situ system for neuronal differentiation and polarity is to measure cortical neurons migration using in utero electroporation. As cortex formation proceeds, newborn neurons in the ventricular zone (VZ) migrate radially to reach the cortical plate. Using in utero electroporation experiments it was described that the EC proteins Exo70 and Exo84 harm neuronal migration [33, 34]. We next investigated a possible role of Sec3 in cortical migration by using in utero electroporation of cortical progenitors at embryonic day (E) 15 to visualize neurons destined to comprise layers II-IV of the cortex, allowing analysis of the location and morphology of the progeny after 3 days of in situ development. The co-electroporation of shRNA-Sec3 and a plasmid encoding red fluorescent protein (DsRed) resulted in effective suppression of Sec3 expression in most electroporated cells, as shown in Fig.4. In brains co-electroporated with a non-relevant shRNA control (ssCtrl) and DsRed plasmid, and then immunostained with antibodies to Sec3 (Fig. 4-bottom), almost all the electroporated cells showed evident staining, essentially no cells electroporated with the shRNA-Sec3 (Fig. 4 -top, left) were stained.

We next analyzed the differentiation of cells at E18. At E18, about 5\% of the DsRed neurons electroporated with scrambled sequence (ssCtrl) were located in the 
ventricular zone/subventricular zone (VZ/SVZ), 35\% of cells were found migrating through the IZ and most neurons (over 60\%) had reached the top of the cortical plate (Fig.5 a-left; quantification shown in Fig.5 b). Neurons not expressing Sec3 showed a different migration pattern at E18. Over $80 \%$ of the cells remained arrested at the VZ/SVZ and IZ compared to less than $40 \%$ in the control experiments and less than $20 \%$ reached the top of the cortical plate compared to $60 \%$ in the control brains (Fig.5 a, middle, quantification shown in Fig. 5 b). To discard the possibility of off-target effects of the shSec3, we coelectroporated brains with Sec3 shRNA- plus a cDNA encoding for human Sec3 (Sec3FL). The results of this experiment showed that co-electroporation with Sec3FL recovered migration to near control levels, with over $40 \%$ of the cells reaching layers II-IV compared to less than $20 \%$ in the brains electroporated with shRNA-Sec3 alone (Fig.5 a, right. quantification shown in Fig. 5 b).

Sec3 is necessary for the polarity switch in cortical neurons

During radial migration of cortical neurons many multipolar neurons can be observed at the lower intermediate zone. This cells extend and retract thin processes at random directions not following the radial glia [35-37]. After this stage, as cells approach the middle of the intermediate zone one neurite grows faster to form the leading process (the future apical dendrite), followed by the appearance of a trailing process (the future axon), acquiring a bipolar morphology radial glial migration [38]. After the axon emerges, the centrosomes and Golgi of the neurons get reoriented toward the cortical plate surface, as they move to the upper part of the intermediate zone [39]. We next analyzed if the EC protein Sec3 participates in the early transition from multipolar to bipolar morphology. Animals were electroporated at E15 and observed at E17. At this time, previous studies have shown that most cells in the in the upper intermediate zone and cortical plate are bipolar [40, 41]. Our results show that in control conditions around $80 \%$ of the neurons migrating through the 
middle intermediate zone exhibited a bipolar morphology, as defined by the absence of more than two projections (Fig 6 a Top, arrow, quantification shown in Fig. 6 b). In contrast around $70 \%$ of the shRNASec3 electroporated cells were arrested as multipolar cells (Fig. 6 a, middle top, arrow, quantification shown in Fig 6 b). To discard the possibility of nonspecific or off-target effects of the shSec3, we co-electroporated brains with shSec3 plus a cDNA encoding for full length human Sec3 (Sec3FL). The results of this experiment showed that co-electroporation with $\operatorname{shSec3/Sec3FLshowed~an~increase~in~the~neurons~}$ displaying a bipolar morphology close to control level (Fig.6 a bottom, right. quantification shown in Fig. 6 b). This demonstrates that the multipolar/bipolar switch is diminished when Sec3expression is suppressed.

\section{Discussion}

The last years a lot of effort had been dedicated to the study of the exocyst complex structure. It has been recently published that the first step in the exocyst complex formation involves the formation of two subcomplexes: i) The I subcomplex containing Sec3, Sec5, Sec6 and Sec8, and ii) The II subcomplex holding Sec10, Sec15, Exo 70 and Exo84. After contacting the plasmalemma this two subcomplexes fit together forming the whole exocyst complex to anchor the vesicle to the plasmalemma. Before fusion occurs, the Sec3 subunit is liberated from the exocyst complex. After fusion has started, the remaining exocyst subunits get freed from the fusion point. A remarkable concept raised from these examinations, indicates that although Sec3 is necessary to form the exocyst complex, this subunit is softly bond to the complex and gets liberated before fusion [27]. Also some interesting interactions of Sec3 have been mapped at the level of specific residues, indicating that the N-terminal part of this protein attaches to the SNARE Sso1 
increasing the rate for SNARE complex formation [42]. It has been also shown a direct interaction between Sec3 and the small GTPase Rho1 in yeast [16]. Moreover, it has been described that Rho GTPases are fundamental not only for cytoskeleton remodeling but also for the establishment of cell polarity. In numerous tissues and organs, including the CNS, this protein family plays a pivotal role in cell migration, looking good as a probable candidate for involvement in neuronal migration disorders [43]. All this information suggests that Sec3 may have a regulatory role in the exocyst dynamics and, consequently, in exocytosis.

Many findings point to the EC as important for polarized delivery in several cell types [44]. For example, the yeast EC marks regions of new membrane addition during budding and cytokinesis $[33,45,46]$. In multi-cellular organisms the EC has been also involved in different processes including exocytosis, such as the establishment of polarity in epithelial cells [13], the insertion of Glut4, the glucose transporter, into the plasmalemma of adipocytes [47] and postsynaptic NMDA and AMPA receptor trafficking in dendrites [48] . Developing neurons necessitate to add a significant amount of newly synthetized membrane to their plasmalemma to support axonal formation and elongation. This is achieved by exocytosis of PPVs specially at the axonal growth cone. We have previously published that PPV's exocytosis is triggered by IGF-1 at the third distal axon and the axonal growth cone [4]. The activation of the small GTPase TC10 and the exocyst component Exo70 is also involved in this pathway required for the control of plasmalemmal enlargement [20]. Moreover, the yeast exocyst marks regions of membrane addition during budding and cytokinesis [33, 45, 46]. An NGF-induced Exo70TC10 complex has been shown to modulate neurite outgrowth in PC12 cells [23]. Beside these data, no functional studies about the function of individual components of the 
exocyst complex on neuronal differentiation and establishment of neuronal polarity have been yet performed.

The goal of the experiments shown in the present report was to determine the possible participation of the exocyst protein $\operatorname{Sec} 3$ on the regulation of axonal initial outgrowth necessary for the establishment of neuronal polarity. We first investigated the temporal expression of Sec3 in developing neurons showing that the former is expressed early in neurons in stages 1 and 2 before polarization and located preferentially at distal axons and growth cones. Silencing experiments using Sec3-targeted shRNA indicated that this exocyst protein is necessary for the regulation of initial axonal outgrowth and hippocampal neuron polarization. Next, we studied in situ the role of Sec3 on migration and differentiation of pyramidal cortical neurons by in utero electroporation. During brain cortex formation, the migrating young neurons transiently exhibit a multipolar morphology at the VZ/SVZ region, at the time the neurons penetrate the IZ the cell morphology changes and the neurons become bipolar, with a leading process (the future axial dendrite) and a training process (the future axon) $[35,36,40,41]$. This change in morphology is an essential step in neuron migration indispensable for the definition of neuronal subtypes and the accurate lamination of the neocortex [36, 49-52]. Our results show that loss of function of Sec3 (see above) maintain most of the neurons as multipolar, inhibiting the polarity change at the IZ. It follows that most neurons with knocked-down expression of Sec3 remain arrested at the VZ/SVZ/IZ and are unable to form an axon, abolishing neuronal polarity. Co-transfection whit a human form of Sec3 rescued the morphology allowing near to normal migration and differentiation.

Although our study focused on Sec3, the exocyst consists of one copy of each of its eight subunits (845 kDa in total): Sec3, Sec5, Sec6, Sec8, Sec10, Sec15, Exo70, and 
Exo84 [46, 53, 54]. In spite that the ideas about the dynamics of the formation of the exocyst complex have change along the years, Sec3 protein has always been located in contact with the plasma membrane as well as the exocyst partner Exo70 [17, 45, 55-57]. With the following results, we have uncovered a role of Sec3 in the regulation of neuronal polarity. In the present work, using primary cultures and in situ electroporation, we are able for the first time to broaden the knowledge of the role of the EC in neurons and sum up to previous data from our laboratory that demonstrates that Exo70 also is needed to neuronal polarity. Intriguingly both proteins, Sec3 and Exo70, are located at the plasma membrane. More investigation will be needed in order to determine the precise roles of all the exocyst proteins on neuronal differentiation and polarization and if its localization at the exocyst complex define its role in the establishment of polarity.

\section{Materials and methods}

DNA Constructs The validated shRNA plasmids were purchase to Sigma ( Aldrich, RRID: SCR_008988) under the following label name shSec348-Clone ID: NM_027270.1-2811s21c1, shSec345-Clone ID: NM_027270.1-2716s21c1. Control shRNA, refered as ssCtrl does not recognize any mouse or rat sequence. All the shRNA plasmids were clone into a pLKO.1 CMV-tGFP. The construct pCAG-DsRed was a generous gift from Connie Cepko. The construct Sec3-GFP was cloned into a pEGFP-N3 vector (RRID: Addgene_62043).

Antibodies. The following primary antibodies were used: rabbit polyclonal antibody to

Sec3 (Abcam Cat\# ab118798, RRID:AB_10900337, diluted 1/700 for immunofluorescence (IF)) and 1:1000 for Western blot (WB); mouse monoclonal antibody to tyrosinated tubulin (SIGMA Cat. T-90268), diluted to 1:1000 (IF); mouse monoclonal antibody to the axonal marker Tau-1 (Millipore Cat\# MAB3420, RRID: 
AB_94855]), diluted to 1:600 (IF) rabbit polyclonal antibody to phosphorylated IGF1r (Cell Signaling Cat. C14A11 diluted to 1:60 (IF)) and mouse monoclonal antibody to $\alpha$ tubulin (Thermo Fisher Scientific Cat\# 62204, RRID: AB_1965960), diluted to 1:2000 (WB). The corresponding secondary antibodies for fluorescence microscopy were Alexa Fluor 488, 568 and 633 (Thermo Fisher Scientific, anti-rabbit-AF488 cat. A-21441, antimouse-AF594 cat. \#A11077, anti-rabbit-AF594 cat. \#A11077 and anti-mouse-AF633 cat. \#A11077).

Animals All animal procedures were done according to the "Guide for the care and use of laboratory animals" (8 th Edition, USA), Federation of European Laboratory Animal Science Associations (FELASA) and approved by of the Institutional Board for Animal Welfare (CICUAL, Facultad de Ciencias Químicas, Universidad Nacional de Córdoba. Approval number 747/2018, UNC: 0002349/2018 and 1003/2018, UNC: 001 5868/2018). Time-pregnant C57BL/6CIQBC mice were provided by our facility. Timepregnancies were achieved in animals from 2 to 6 months of age. Animals were under standard cages and companion, ad libitum access to food and water, with a 12-hour light/dark cycle and controlled humidity $(40-70 \%)$ and temperature $\left(20-26^{\circ} \mathrm{C}\right)$. At least 3 female rats or mouse were used to carry on each experiment. Each female animal is treated as an independent experiment.

Cell culture. Hippocampal culture and transfection Dissociated hippocampal pyramidal neurons were prepared from fetal mouse brain and cultured as described previously in [58]. Briefly, pregnant C57BL/6CIQBC mice were asphyxiated by $\mathrm{CO}_{2}$ at 18 days postfertilization and subsequent cervical dislocation. The embryos were recovered creating an opening in the mid ventral side of the mouse with large sterile surgical scissors removed from their individual placenta and then decapitated with sterile scissors in a 
laminar flow hood. The removed heads were placed in plates with sterile Hank's Balance Salt Solution (HBSS, Sigma-Aldrich, RRID:SCR_008988) at $4^{\circ} \mathrm{C}$ under a dissecting microscope in order to remove the brain, grasp the meninges and remove the hippocampus. Isolated hippocampus were enzymatically dissociated with trypsin $(0.25 \%$ w/v for $18 \mathrm{~min}$ at $37^{\circ} \mathrm{C}$ Life Technologies, RRID: SCR_008817) followed by mechanical trituration with fire polished Pasteur pipettes. After resuspension the cells were plated

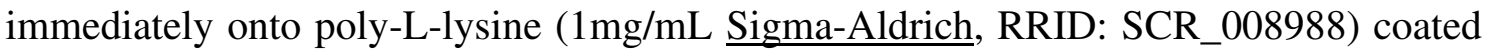
glass coverslips and maintained in Dulbecco's modified Eagle's medium (DMEM, Gibco Thermo Fisher Scientific, RRID: SCR_008452) in a rich serum environment completed by adding 10\% horse serum (Gibco Thermo Fisher Scientific) for $2 \mathrm{~h}$. Neurons are weaned to serum-free Neurobasal medium with N2 and B27 supplements (Thermo Fisher Scientific, RRID: SCR_008452). For those experiments involving expression of shRNA sequences in early stages of neuronal development, a protocol of transfection of neurons in suspension, before plating, was used, similar to the procedure described in [59] with minor modifications. Shortly, two different solutions were prepared; one with the DNA and the other with the Lipofectamine. As general rule, for one coverslip the solution with Lipofectamine $^{\mathrm{TM}} 2000$ Transfection Reagent (Thermo Fisher Scientific, RRID: SCR_008452) is diluted in Opti-MEM (Gibco Thermo Fisher Scientific, RRID: SCR_008452) in a relation of $1.2 \mu \mathrm{l}$ of Lipofectamine per $48 \mu \mathrm{l}$ of Opti-MEM. On the other hand, $0.6 \mu \mathrm{g}$ of DNA and $30 \mu \mathrm{l}$ of Opti-MEM are mixed. Both mixtures (80 $\mu \mathrm{l})$ are incubated together at room temperature for $30 \mathrm{~min}$ and after that 70.000 cells, previously diluted in an indicated volume of Opti-MEM, were added. Cells-DNA-Lipofectamine mixture was immediately plated over poly-L-lysine -coated glass coverslips and cultures were placed to $37{ }^{\circ} \mathrm{C}$ in a humidified $5 \% \mathrm{CO}_{2}$ incubator. After $1.15 \mathrm{~h}$, transfecting complex was removed carefully from each coverslip (at this time most of neurons were 
already attached) and serum-free medium plus the N2 mixture was added to cultures. Cultures were placed in a humidified $37{ }^{\circ} \mathrm{C}$ incubator with $5 \% \mathrm{CO}_{2}$ for $24-48 \mathrm{~h}$ before fixation. Shortly after plating, hippocampal neurons first extend lamellipodia (stage 1) and afterward several minor neurites that are initially indistinguishable (stage 2). Then, at stage 3 , one of these initially equivalent neurites grows more rapidly than the others and becomes the axon, whereas the other neurites subsequently develop into dendrites (stage 4). Neurons are considered to be at stage 3 when the length of the axon exceeds that of the average minor neurite by at least $20 \mu \mathrm{m}$. Line cell culture. For sh validation frozen vials of Neuro-2a mus Musculus (ATCC® CCL-131 ${ }^{\mathrm{TM}}$ ) were recovered. Cells were cultured in DMEM (Dulbecco's modified Eagle medium) supplemented with 10\% of fetal bovine serum (FBS) (Gibco Thermo Fisher Scientific) and maintained in a humidified $37{ }^{\circ} \mathrm{C}$ incubator with $5 \% \mathrm{CO}_{2}$. Transient transfection of cultured neurons was performed as described previously, and the constructs used at concentration of $1.5 \mu \mathrm{g}$ or $2.5 \mu \mathrm{g}$ per $60 \mathrm{~mm}$ dish [60].

Isolation of growth cones Axonal growth cones were isolated from developing brain as described previously [40, 41]. In brief, brains of 18 days post-fertilization embryos were homogenized (H). A low-speed supernatant (LSS) was prepared, loaded onto a discontinuous sucrose density gradient with steps of $0.83 \mathrm{M}, 1 \mathrm{M}$ and $2.66 \mathrm{M}$ sucrose, and spun to equilibrium at $242,000 \mathrm{~g}$. The fraction at the $10 \mathrm{~d} / 0.83 \mathrm{~m}$ interface (designated "A") contained the isolated growth cones or growth cone particles (GCPs).

Immunofluorescence microscopy. Neurons were fixed for $20 \mathrm{~min}$ at room temperature in phosphate-buffered saline (PBS) solution containing paraformaldehyde $(4 \% \mathrm{w} / \mathrm{v}$ Sigma-Aldrich, RRID: SCR_008988) and sucrose (4\% w/v). After a thorough washing, the cultures were permeabilized with Triton X-100 (0.2\% v/v Sigma-Aldrich, RRID:SCR_008988) in PBS for 6 min. A 1 hour blocking step at room temperature was 
done before labeling with primary antibodies (overnight at $4{ }^{\circ} \mathrm{C}$ ). After subsequent washes with PBS, cultures were incubated with fluorescent secondary antibodies conjugated to Alexa Fluor 488, 546 or 633 ( $1 \mathrm{~h}$ at room temperature). Coverslips were mounted in slides.

Gel Electrophoresis and Western Blot. Proteins levels were assessed by SDSpolyacrylamide gel electrophoresis (SDS-PAGE). The resolving gel mixture selected was $10 \%$ for all the blots presented in the present work. The resolved proteins were electrotransferred to nitrocellulose membranes (Amersham Hybond-ECL, GE Healthcare Amersham Biosciences, RRID: SCR_013566) in Tris-glycine buffer containing methanol (20\% v/v). The membranes were rinsed with Tris-buffered saline (TBS) (Tris, $\mathrm{pH} 7.510$ $\mathrm{mM}, \mathrm{NaCl} 150 \mathrm{mM}$ ) and 1 hour blocking step at room temperature in TBS containing 5 $\%$ w/v nonfat milk was done before labeling with primary antibodies (overnight at $4{ }^{\circ} \mathrm{C}$ in TBS containing $1 \% \mathrm{w} / \mathrm{v}$ nonfat milk). After subsequent washes with TBS containing Tween $20(0.05 \% \mathrm{v} / \mathrm{v})$, the membranes were incubated with fluorescent Odyssey IRdye CW 800 secondary antibodies (LI-COR Biosciences, LI-COR Biosciences Cat\# 92634211, RRID: AB_1850017, LI-COR Biosciences Cat\# 926-34210, RRID: AB_1850016) secondary antibodies for $1 \mathrm{~h}$ at room temperature. After washing, the blots were exposed to IR light using an Odyssey Infrared Imaging System (Odyssey CLx, RRID: SCR_014579, LI-COR Biosciences).

In utero electroporation (IUE). In utero electroporation was performed as previously described [61] with minor modifications. Briefly, C57BL/6CIQBC mice pregnant at 15 days post fertilization were anaesthetized with isoflurane (Piramal UK). Needles for injection were pulled from P-97 Flaming/Brownglass capillaries (World Precision Instruments, RRID: SCR_008593). The embryonic ventricle was injected with a mixture 
of shRNA/Dsred plasmids $(2 \mu \mathrm{g} / \mu \mathrm{l}-1 \mu \mathrm{g} / \mu \mathrm{l}$ respectively) in sterile water and Tripan blue dye. Five pulses of 38V (50 ms ON, $950 \mathrm{~ms}$ OFF) were applied using $5 \mathrm{~mm}$ electrodes and a dedicated electroporator (LIADE National University of Córdoba, Argentina). The embryos were placed back into the abdominal cavity and the abdominal cavity was sutured. After this procedure, an analgesic, tramadol $(5 \mathrm{mg} / \mathrm{kg}$. Finadiet), was administered. Female mice were allowed to recover from anesthesia on a warm plate.

Immunohistochemistry. 18 days post fertilization an also 3 days after IUE, mice were anesthetized with Ketamine/Xilancine (Holliday/Richmond) mixture. The embryo brains were removed and post-fixed in paraformaldehyde $(4 \%$ w/v $\underline{\text { Sigma-Aldrich, }}$ RRID:SCR_008988) at $4{ }^{\circ} \mathrm{C}$ overnight. The brains were cryoprotected by overnight immersion in sucrose (30\% w/v in PBS) and embedded in OCT. Then the brains were cryopreserved using liquid nitrogen and store at $-80{ }^{\circ} \mathrm{C}$ for two days. Floating coronal cryosections of $20 \mu \mathrm{m}$ were obtained and permeabilized with Triton-X $100(0.5 \% \mathrm{v} / \mathrm{v}$ in PBS) and blocked with BSA and Triton X-100 (2\% w/v and 0.3\% v/v respectively in PBS). The sections were incubated overnight at $4{ }^{\circ} \mathrm{C}$ with primary antibodies and washed with PBS then incubated with Alexa 488 (Molecular Probes Cat\# A-11094, RRID: AB_221544) (1 h at room temperature) and washed with PBS.

Confocal imaging. Images were acquired with the spectral version of Olympus confocal microscope: Fluoview 1200 (Tokyo, Japan) with a $1024 \times 1024$ pixels resolution. For in vitro experiments fluorophores like GFP or Alexa 488 were excited employing a solid state laser line $(473 \mathrm{~nm})$ and its fluorescence collected between 495-540 $\mathrm{nm}$ using oilimmersion objectives lens either a supercorrected 60× PLAPON60XOSC2 1.4 NA; or 40x PLAPON 1.35 NA. For in situ experiments the acquisition were done using low magnifications dry objective lens like 10x UPLANSAPO 0,4NA, 20x UPLANSAPO 0,75 
NA or 40X UPLANSAPO 0,9. For 3D z-stack 10-15 confocal sections of were collected every $2 \mu \mathrm{m}$ step size. Images were analyzed using FIJI software (NIH, MD, USA). All images were processed, and the graphic art was prepared using Adobe PhotoShop (Adobe Photoshop 13.0 version 6.1, RRID: SCR_014199 Adobe Systems, San Jose, CA, USA).

Statistical Analysis and Study design. Statistical analyzes were performed using GraphPad Prism software (GraphPad Prism version 5.00 for Windows, GraphPad Software, San Diego California USA GraphPad Prism RRID: SCR_002798). Data are presented as mean \pm SEM or mean $\pm \mathrm{SD}$, as indicated in the figure legends. In all the cases at least 3 independent experiments from different female rats/mouse were carried out. The in vitro experiments were performed quantifying 100 cells of each set of experiments and in the in situ experiments at least 30 brain slices from 2-3 pups were quantified. Stage assessment in the in vitro experiments was made by considering the length of the axons and Tau1 positive marks. For colocalization analysis Pearson, Manders and Fractionary Mander's colocalization coefficient M1 (proportion of Sec3colocalized with Tau1) with threshold and fluorescence histogram was achieved using the Fiji software and the JACoP plugin. For in utero studies quantification of the migration was accomplished by dividing the cortex into its main areas: VZ - SVZ, IZ and $\mathrm{CP}$ and counting the number of cells in each of these subdivisions. Measurements were normalized to cell number per slice by percentage. Neurons form the upper IZ and CP were considered for morphological analysis considering the maximal projections images from individualized neurons to reconstruct their whole extent. The statistical analysis applied was unpaired t-Test with 95\% confidence interval and two-way ANOVA Test with Bonferroni post-test as indicated in each trial. In all cases: * $\mathrm{p}<0.05, * * \mathrm{p}<0.01$, *** $\mathrm{p}<0.001$, ns: non-significant. The study design was not pre-registered. No randomization was performed to allocate subjects in this study. No blinding was 
performed. No sample calculation was performed; the maximum number of subjects possible was used in each study. We did not run tests to identify outliers and no data points were excluded from the analysis.

\section{References}

1. Pfenninger, K.H. and M.F. Maylie-Pfenninger, Lectin labeling of sprouting neurons. II. Relative movement and appearance of glycoconjugates during plasmalemmal expansion. J Cell Biol, 1981. 89(3): p. 547-59.

2. Futerman, A.H. and G.A. Banker, The economics of neurite outgrowth--the addition of new membrane to growing axons. Trends Neurosci, 1996. 19(4): p. 144-9.

3. Pfenninger, K.H. and L.B. Friedman, Sites of plasmalemmal expansion in growth cones. Brain Res Dev Brain Res, 1993. 71(2): p. 181-92.

4. Pfenninger, K.H., et al., Regulation of membrane expansion at the nerve growth cone. J Cell Sci, 2003. 116(Pt 7): p. 1209-17.

5. Laurino, L., et al., PI3K activation by IGF-1 is essential for the regulation of membrane expansion at the nerve growth cone. J Cell Sci, 2005. 118(Pt 16): p. 3653-62.

6. Quiroga, S., M. Bisbal, and A. Caceres, Regulation of plasma membrane expansion during axon formation. Dev Neurobiol, 2018. 78(3): p. 170-180.

7. Jahn, R., T. Lang, and T.C. Sudhof, Membrane fusion. Cell, 2003. 112(4): p. 519-33.

8. Wickner, W. and R. Schekman, Membrane fusion. Nat Struct Mol Biol, 2008. 15(7): p. 658-64.

9. Lepore, D.M., L. Martinez-Nunez, and M. Munson, Exposing the Elusive Exocyst Structure. Trends Biochem Sci, 2018. 43(9): p. 714-725.

10. Whyte, J.R. and S. Munro, Vesicle tethering complexes in membrane traffic. J Cell Sci, 2002. 115(Pt 13): p. 2627-37.

11. TerBush, D.R., et al., The Exocyst is a multiprotein complex required for exocytosis in Saccharomyces cerevisiae. EMBO J, 1996. 15(23): p. 6483-94.

12. Hsu, S.C., et al., The exocyst complex in polarized exocytosis. Int Rev Cytol, 2004. 233: p. 243-65.

13. Grindstaff, K.K., et al., Sec6/8 complex is recruited to cell-cell contacts and specifies transport vesicle delivery to the basal-lateral membrane in epithelial cells. Cell, 1998. 93(5): p. 731-40.

14. Hazuka, C.D., et al., The sec6/8 complex is located at neurite outgrowth and axonal synapse-assembly domains. J Neurosci, 1999. 19(4): p. 1324-34.

15. Martin-Urdiroz, M., et al., The Exocyst Complex in Health and Disease. Front Cell Dev Biol, 2016. 4: p. 24.

16. Munson, M. and P. Novick, The exocyst defrocked, a framework of rods revealed. Nat Struct Mol Biol, 2006. 13(7): p. 577-81.

17. Picco, A., et al., The In Vivo Architecture of the Exocyst Provides Structural Basis for Exocytosis. Cell, 2017. 168(3): p. 400-412 e18.

18. Mei, K., et al., Cryo-EM structure of the exocyst complex. Nat Struct Mol Biol, 2018. 25(2): p. 139-146. 
19. Mei, K. and W. Guo, Exocytosis: A New Exocyst Movie. Curr Biol, 2019. 29(1): p. R30-R32.

20. Dupraz, S., et al., The TC10-Exo70 complex is essential for membrane expansion and axonal specification in developing neurons. J Neurosci, 2009. 29(42): p. 13292-301.

21. Vega, I.E. and S.C. Hsu, The exocyst complex associates with microtubules to mediate vesicle targeting and neurite outgrowth. J Neurosci, 2001. 21(11): p. 3839-48.

22. Lalli, G. and A. Hall, Ral GTPases regulate neurite branching through GAP-43 and the exocyst complex. J Cell Biol, 2005. 171(5): p. 857-69.

23. Pommereit, D. and F.S. Wouters, An NGF-induced Exo70-TC10 complex locally antagonises $C d c 42-m e d i a t e d$ activation of $N$-WASP to modulate neurite outgrowth. J Cell Sci, 2007. 120(Pt 15): p. 2694-705.

24. Lalli, G., RalA and the exocyst complex influence neuronal polarity through PAR-3 and aPKC. J Cell Sci, 2009. 122(Pt 10): p. 1499-506.

25. Das, A., et al., RalA promotes a direct exocyst-Par6 interaction to regulate polarity in neuronal development. J Cell Sci, 2014. 127(Pt 3): p. 686-99.

26. Murthy, M., et al., Mutations in the exocyst component Sec5 disrupt neuronal membrane traffic, but neurotransmitter release persists. Neuron, 2003. 37(3): p. 433-47.

27. Ahmed, S.M., et al., Exocyst dynamics during vesicle tethering and fusion. Nat Commun, 2018. 9(1): p. 5140.

28. Oda, Y., et al., Novel coiled-coil proteins regulate exocyst association with cortical microtubules in xylem cells via the conserved oligomeric golgi-complex 2 protein. Plant Cell Physiol, 2015. 56(2): p. 277-86.

29. Dotti, C.G., C.A. Sullivan, and G.A. Banker, The establishment of polarity by hippocampal neurons in culture. J Neurosci, 1988. 8(4): p. 1454-68.

30. Lohse, K., et al., Axonal origin and purity of growth cones isolated from fetal rat brain. Brain Res Dev Brain Res, 1996. 96(1-2): p. 83-96.

31. Pfenninger, K.H., et al., Nerve growth cones isolated from fetal rat brain: subcellular fractionation and characterization. Cell, 1983. 35(2 Pt 1): p. 573-84.

32. Sosa, L., et al., IGF-1 receptor is essential for the establishment of hippocampal neuronal polarity. Nat Neurosci, 2006. 9(8): p. 993-5.

33. Guo, W., A. Grant, and P. Novick, Exo84p is an exocyst protein essential for secretion. J Biol Chem, 1999. 274(33): p. 23558-64.

34. Letinic, K., et al., Exocyst is involved in polarized cell migration and cerebral cortical development. Proc Natl Acad Sci U S A, 2009. 106(27): p. 11342-7.

35. Tabata, H. and K. Nakajima, Multipolar migration: the third mode of radial neuronal migration in the developing cerebral cortex. J Neurosci, 2003. 23(31): p. 9996-10001.

36. Noctor, S.C., et al., Cortical neurons arise in symmetric and asymmetric division zones and migrate through specific phases. Nat Neurosci, 2004. 7(2): p. 136-44.

37. Bielas, S., et al., Cortical neuronal migration mutants suggest separate but intersecting pathways. Annu Rev Cell Dev Biol, 2004. 20: p. 593-618.

38. Yogev, S. and K. Shen, Establishing Neuronal Polarity with Environmental and Intrinsic Mechanisms. Neuron, 2017. 96(3): p. 638-650.

39. Gupta, A., L.H. Tsai, and A. Wynshaw-Boris, Life is a journey: a genetic look at neocortical development. Nat Rev Genet, 2002. 3(5): p. 342-55.

40. Bai, J., et al., RNAi reveals doublecortin is required for radial migration in rat neocortex. Nat Neurosci, 2003. 6(12): p. 1277-83. 
41. Namba, T., et al., Pioneering axons regulate neuronal polarization in the developing cerebral cortex. Neuron, 2014. 81(4): p. 814-29.

42. Yue, P., et al., Sec3 promotes the initial binary t-SNARE complex assembly and membrane fusion. Nat Commun, 2017. 8: p. 14236.

43. Cappello, S., et al., A radial glia-specific role of RhoA in double cortex formation. Neuron, 2012. 73(5): p. 911-24.

44. Novick, P., C. Field, and R. Schekman, Identification of 23 complementation groups required for post-translational events in the yeast secretory pathway. Cell, 1980. 21(1): p. 205-15.

45. Finger, F.P., T.E. Hughes, and P. Novick, Sec3p is a spatial landmark for polarized secretion in budding yeast. Cell, 1998. 92(4): p. 559-71.

46. TerBush, D.R. and P. Novick, Sec6, Sec8, and Sec15 are components of a multisubunit complex which localizes to small bud tips in Saccharomyces cerevisiae. J Cell Biol, 1995. 130(2): p. 299-312.

47. Inoue, M., et al., The exocyst complex is required for targeting of Glut4 to the plasma membrane by insulin. Nature, 2003. 422(6932): p. 629-33.

48. Sans, N., et al., NMDA receptor trafficking through an interaction between PDZ proteins and the exocyst complex. Nat Cell Biol, 2003. 5(6): p. 520-30.

49. Evsyukova, I., et al., Cleavage and polyadenylation specificity factor 1 (CPSF1) regulates alternative splicing of interleukin 7 receptor (IL7R) exon 6. RNA, 2013. 19(1): p. 103-15.

50. Miyoshi, G. and G. Fishell, Dynamic FoxG1 expression coordinates the integration of multipolar pyramidal neuron precursors into the cortical plate. Neuron, 2012. 74(6): p. 1045-58.

51. Ohshima, T., et al., Cdk5 is required for multipolar-to-bipolar transition during radial neuronal migration and proper dendrite development of pyramidal neurons in the cerebral cortex. Development, 2007. 134(12): p. 2273-82.

52. Pacary, E., et al., Proneural transcription factors regulate different steps of cortical neuron migration through Rnd-mediated inhibition of RhoA signaling. Neuron, 2011. 69(6): p. 1069-84.

53. Heider, M.R. and M. Munson, Exorcising the exocyst complex. Traffic, 2012. 13(7): p. 898-907.

54. Hsu, S.C., et al., Targeting vesicles to specific sites on the plasma membrane: the role of the sec6/8 complex. Trends Cell Biol, 1999. 9(4): p. 150-3.

55. Boyd, C., et al., Vesicles carry most exocyst subunits to exocytic sites marked by the remaining two subunits, Sec3p and Exo70p. J Cell Biol, 2004. 167(5): p. 889-901.

56. Dong, G., et al., The structures of exocyst subunit Exo70p and the Exo84p Cterminal domains reveal a common motif. Nat Struct Mol Biol, 2005. 12(12): p. 1094-100.

57. Yamashita, M., et al., Structural basis for the Rho- and phosphoinositidedependent localization of the exocyst subunit Sec3. Nat Struct Mol Biol, 2010. 17(2): p. 180-6.

58. Banker, G.A. and W.M. Cowan, Rat hippocampal neurons in dispersed cell culture. Brain Res, 1977. 126(3): p. 397-42.

59. Halterman, M.W., et al., In-tube transfection improves the efficiency of gene transfer in primary neuronal cultures. J Neurosci Methods, 2009. 177(2): p. 348-54.

60. Rosso, S.B., et al., Wnt signaling through Dishevelled, Rac and JNK regulates dendritic development. Nat Neurosci, 2005. 8(1): p. 34-42. 
61. Saito, T. and N. Nakatsuji, Efficient gene transfer into the embryonic mouse brain using in vivo electroporation. Dev Biol, 2001. 240(1): p. 237-46.

\section{Acknowledgments}

We acknowledge the technical and imaging assistance from Dr. Cecilia Sampedro and Dr. Carlos Más, Centro de Micro y Nanoscopía de Córdoba, CEMINCO (CONICET), Universidad Nacional de Córdoba, Córdoba, Argentina and the technical assistance on animal care and maintenance from Rosa Andrada, Gimena Farias and Laura Argañaras (CIQUIBIC, CONICET). We thank Dr. José Luis Barra (CIQUIBIC, CONICET, Universidad Nacional de Córdoba, Córdoba, Argentina) for his counseling in molecular biology experiments.

This work was supported by the Secretaría de Ciencia y Técnica, Universidad Nacional de Córdoba and the Fondo para la Investigación Científica y Tecnológica, Secretaría de Ciencia, Tecnología e Innovación Productiva, Argentina [grant numbers PICT 2013-1646 and PICT 2015-2286 to SQ].

\section{This study was carried out in compliance with the ARRIVE guideliness}

\section{Author contributions}

All authors contributed to the study conception and design. Material preparation, data collection and analysis were performed by FBP. LJS discussed the results and participated in the preparation of the paper. SQ and FBP planned experiments, discussed the results and participated in the preparation of the paper. All authors commented on the manuscript. All authors read and approved the final manuscript.

\section{Competing interests}


Florentyna Bustos Plonka, Lucas J. Sosa and Santiago Quiroga. declare that the authors have no competing interests as defined by Nature Research, or other interests that might be perceived to influence the results and/or discussion reported in this paper.

Figure and Figure Legends

a)

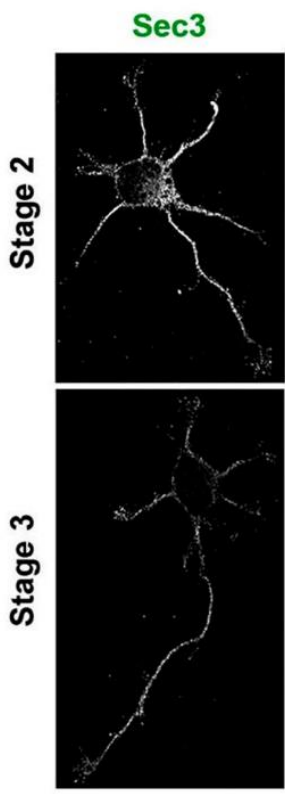

Tubulin
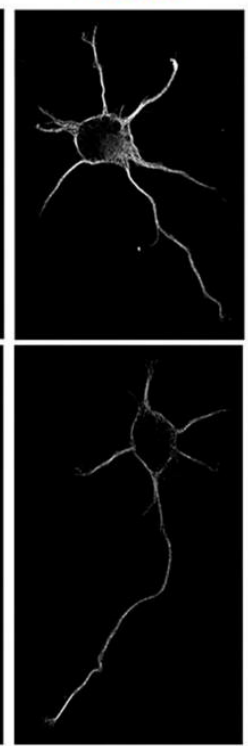

Merge

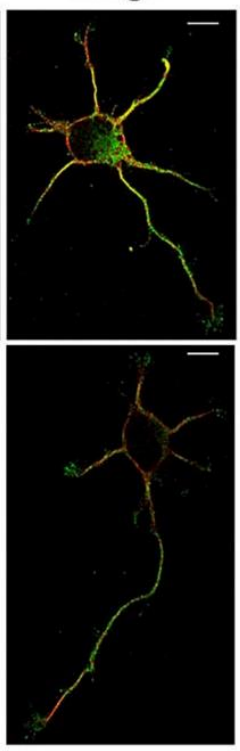

b)

12243672

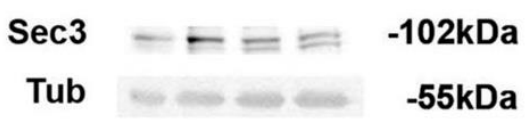

c)

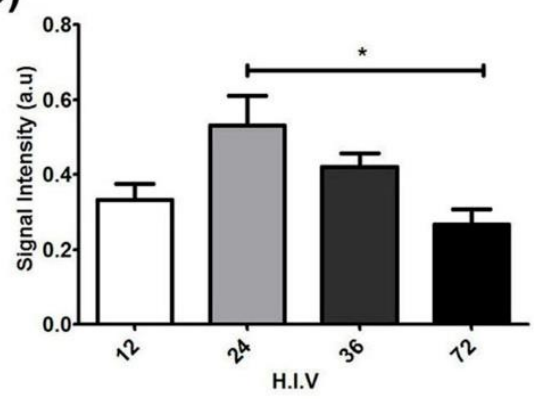

Fig 1-Sec3 is expressed previous to the polarization event, during the polarization and presents a significant diminution when the cells are fully polarized a) Representatives images of double immunofluorescence showing the distribution of Sec3 (green) and tyrosinated tubulin (red) in hippocampal pyramidal neurons in culture at late stage 2 after 18 (top) or stage 3 after 30 (bottom) H.I.V. Calibration bar $=20 \mu \mathrm{m}$. b) Western Blot of hippocampal cell lysates harvested at 12, 24, 36, or 72 H.I.V showing the expression of Sec3 (first row; apparent molecular weight $103 \mathrm{kDa}$ ) and tubulin (second row) as a loading control and secondary antibodies suitable for near infrared fluorescence. The obtained images were converted to grey scale and inverted. The results of one of three independent experiments are shown. The blots belong to the same experiment but were cropped for a better understanding. c) Quantification of Western blot for Sec3. Bars represent mean \pm SEM of the density measured in each band relative to tubulin density. A two-way ANOVA Test with Bonferroni post-test was performed. $* \mathrm{P} \leqslant 0.05 \mathrm{n}=3$ independent experiments. 
a)

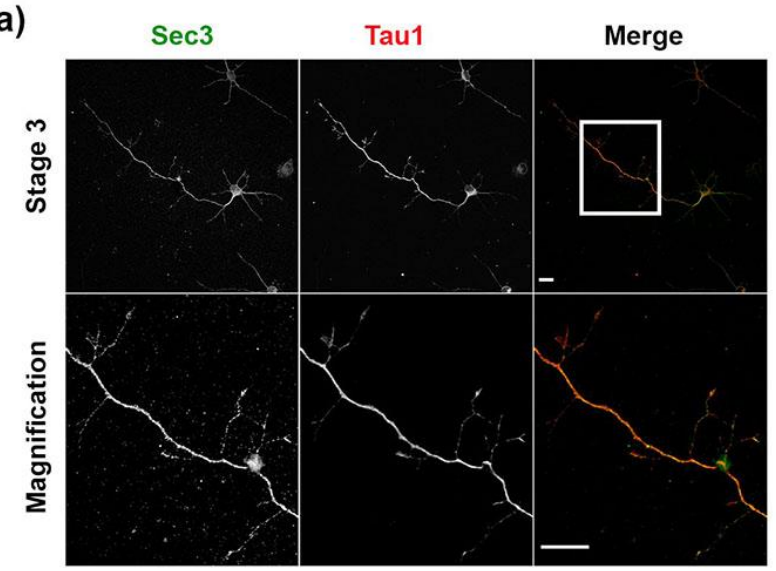

d)

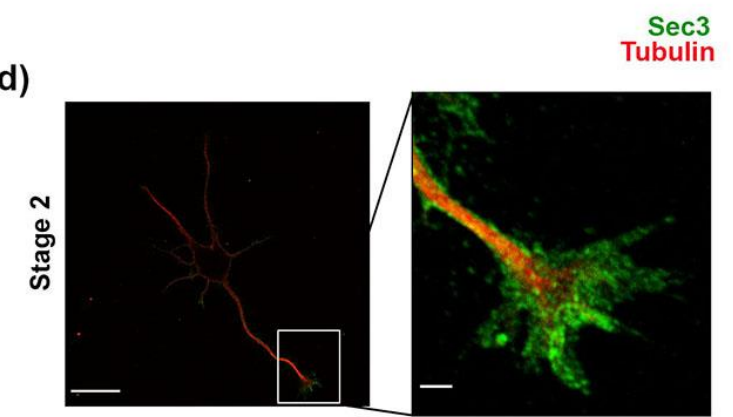

b)
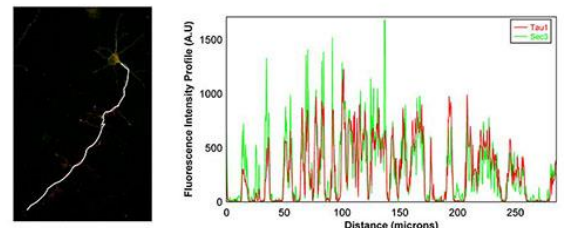

c)

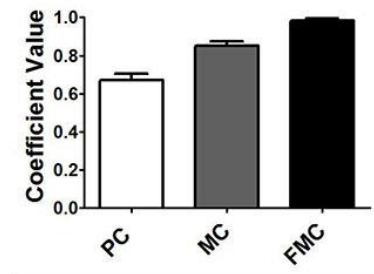

e)

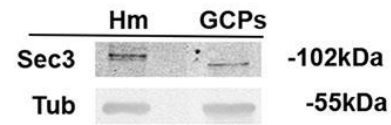

Fig 2-Sec3 is localized at the axon and the growth cone. a) Representative images of double immunofluorescence micrographs showing the localization of Sec3 (green) or Tau1 (red) in hippocampal pyramidal neurons in culture at stage 3 after 24 H.I.V (top). A magnification of the axon is shown (bottom). Note the enriched co-localization at the distal axon. Calibration bar $=20 \mu \mathrm{m}$. b) Histogram (right) showing fluorescence intensity spatial profiles through the white line showing Sec3 (green channel) or and Tau1 (red channel) along white line showed in the merged image (left). c) Quantitative analysis of co-expression levels by Pearson's colocalization coefficient, Manders colocalization coefficient and Fractionary Mander's (M1 coefficient is expressed indicating Sec3 that colocalizes with Tau1 using JACoP, ImageJ. Bars represent mean \pm SEM of the coefficient. $n=3$ independent experiments 10 cells were scored for each coefficient measure. d) Representative images of double immunofluorescence micrographs showing the localization of Sec3 (green) or Tyr Tubulin (red) in axonal growth cone in hippocampal pyramidal neurons in culture at late stage 2 after 18 H.I.V. A magnification of the axon is shown (left). Calibration bar $=20 \mu \mathrm{m}$. e) Western blot of total 18-day rat embryo brain homogenate (first column) and GCPs (second column) showing the expression of Sec3 (first row; apparent molecular weight $103 \mathrm{kDa}$ ) and tubulin (second row) as a loading control and secondary antibodies suitable for near infrared fluorescence. The obtained images were converted to grey scale and inverted. The results of one of three independent experiments are shown. The blots belong to the same experiment but were cropped for a better understanding. 
a)

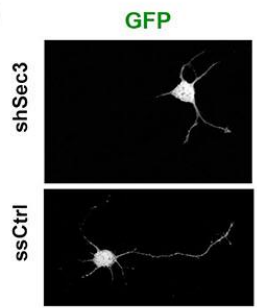

b)

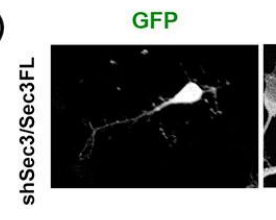

e)

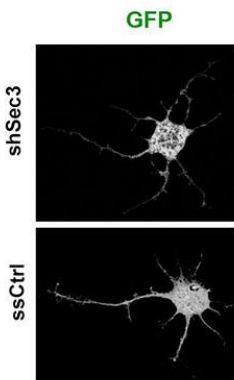

Tau1

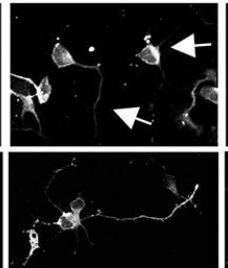

Tau1

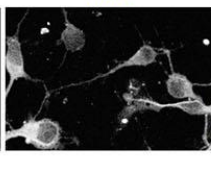

pIGF1

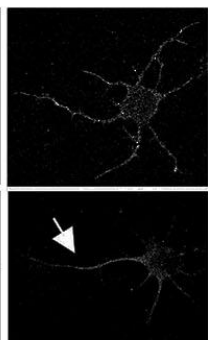

$\operatorname{Sec} 3$

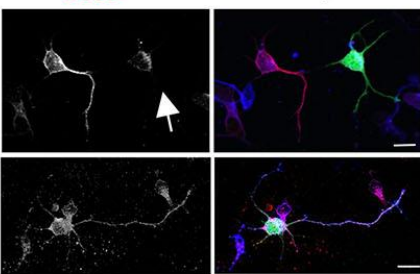

Merge

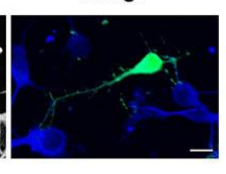

Tyr Tub

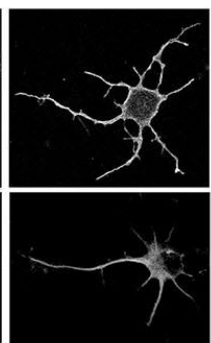

Merge

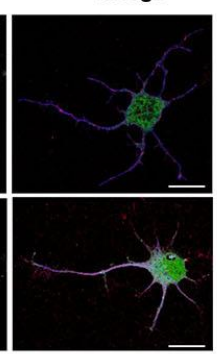

c)

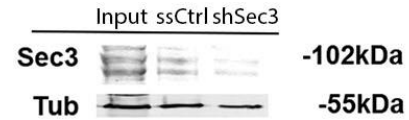

d)

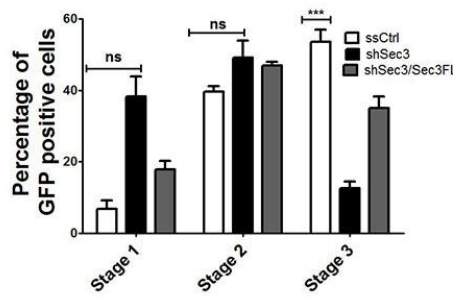

f)

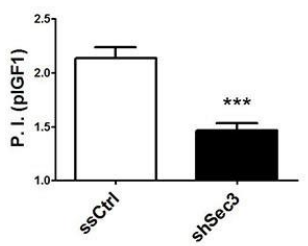

Fig 3 Sec3 is necessary for the establishment of neuronal polarity in mouse hippocampal neurons. a) Representative images of double immunofluorescence micrographs showing the localization of Sec3 (red, third column) or Tau-1 (blue, second column) and GFP as a transfection marker (first column) in hippocampal pyramidal neurons in culture at stage 3 after 24 H.I.V transfected with Sec3-targeted shRNA ( $\operatorname{shSec3}$, first row) and a scrambled RNA sequence (ssCtrl, second row). Note that the neurons transfected with Sec3-targeted shRNA (arrow) did not develop axons and did not target Tau-1 to any particular neurite. Note that cells transfected with an ssCtrl exhibit an axon-like process enriched in Tau-1. Calibration bar $=20 \mu \mathrm{m}$. b) Representative images of double immunofluorescence micrographs showing the localization of Tau-1 (blue, second column) and GFP as a transfection marker (first column) in hippocampal pyramidal neurons in culture at stage 3 after 24 H.I.V transfected with Sec3-targeted shRNA and Sec3FL. Note that the neurons transfected with Sec3-targeted shRNA and Sec3FL develop axons. Calibration bar $=20 \mu \mathrm{m}$. c) Western blots of mouse Neuro-2a harvested at 24 H.I.V showing protein levels in culture in the presence of different Sec3targeted shRNA (first row; apparent molecular weight $103 \mathrm{kDa}$ ) and tubulin (second row) as a loading control and secondary antibodies suitable for near infrared fluorescence. The obtained images were converted to grey scale and inverted. The blots belong to the same experiment but were cropped for a better understanding. d) Quantification of neuronal stages of cells transfected with Sec3-targeted shRNA, Sec3-targeted shRNA with a full length Sec3 protein or control sequence after 24 H.I.V. Bars represent mean \pm SEM of the percentage. A Two-way ANOVA Test with Bonferroni post-test was performed. $* * * \mathrm{P}$ $\leqslant 0.001$, ns: non significance. $\mathrm{n}=3$ independent experiments. At least 100 neurons were scored for each condition. e) Representative images of double immunofluorescence micrographs showing the distributions of phosphorylated IGF-1r (red, second column), tyrosinated tubulin (blue, third column) and the transfection marker GFP (first column) in hippocampal pyramidal neurons in culture at stage 2 after 20 H.I.V transfected with Sec3-targeted shRNA (shSec3, first row) or a scrambled RNA sequence (ssCtrl, second 
row). Neurons were deprived of growth factors for $4 \mathrm{~h}$ and stimulated with $20 \mathrm{nM}$ IGF-1 for $2 \mathrm{~min}$ before fixation. Note the polarization of active (membrane-inserted) IGF-1r to one of the minor neurites of the cell transfected with ssRNA (bottom, arrow). In contrast, neurons transfected with $\mathrm{Sec} 3$ (bottom) failed to polarize the active IGF-1r to any neurite. Calibration bar $=20 \mu \mathrm{m}$. $\mathrm{f}$ ) Quantification of a polarization index of active IGF-1r (P.I. IGF-1r.) calculated as the fluorescence intensity (A.U.) of the brightest minor neurite/average fluorescence intensity (A.U.) of the other minor neurites of the same cell. Bars represent mean \pm SEM of the P.I. Neurons were processed as in c. The polarization index is significantly higher in neurons transfected with scrambled sequence compared to those transfected with shSec3. A t -Test was performed $* * * \mathrm{P} \leq 0.001$. ns: non-significant $\mathrm{n}=3$ independent experiments 10 cells were scored for each condition.

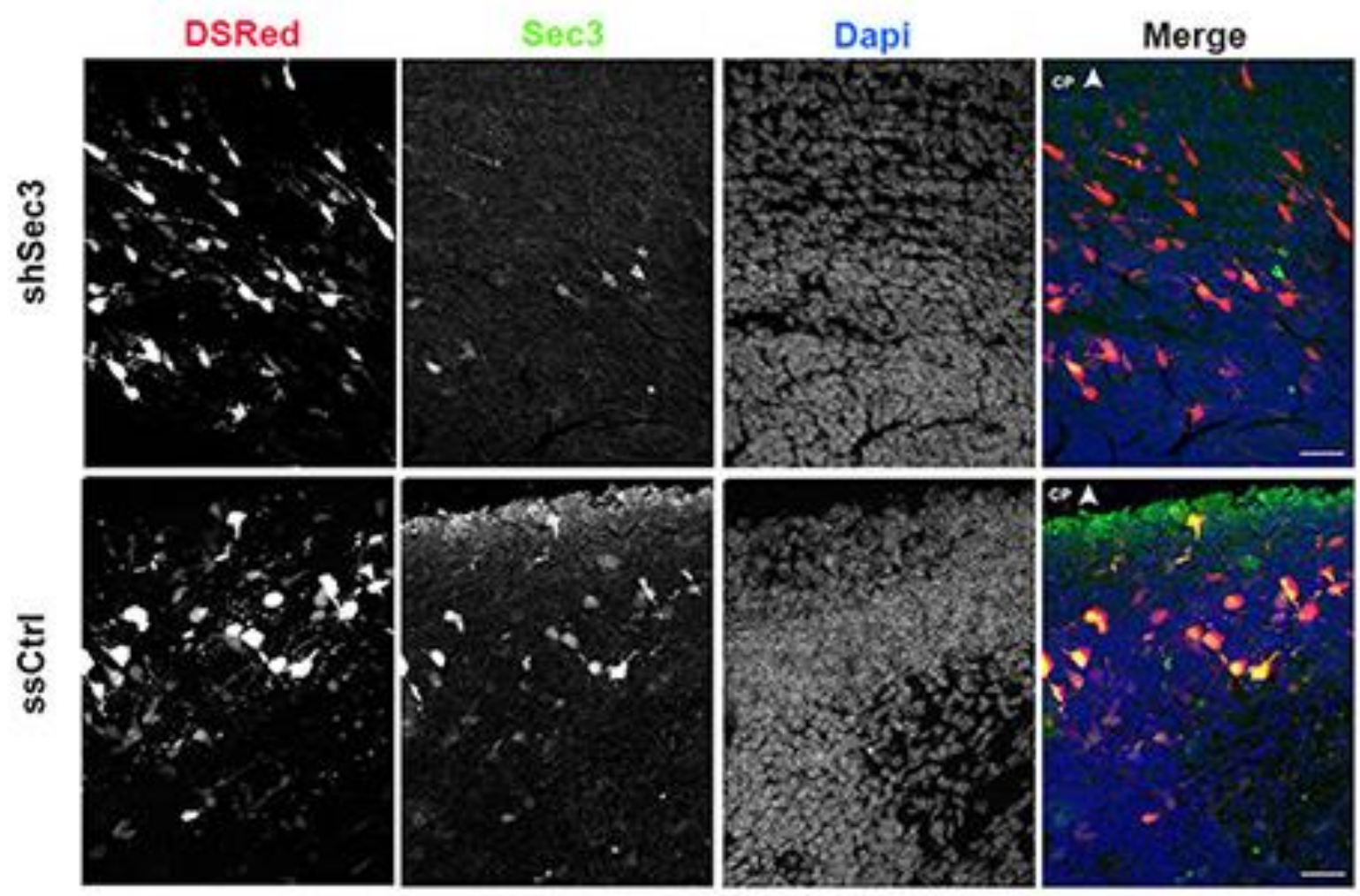

Fig 4-Electroporation with $\operatorname{shSec} 3$ reduces expression Sec3. a) Representative images of brains co-electroporated at E15 and analyzed at E17 with Sec3-targeted shRNA (top) or control ssCtrl (bottom) with DsRed showing the expression of DsRed positive cells (first row), Sec3 (green, second row) and Dapi (third row). The Cortical Plate (CP) is labeled for orientation. 


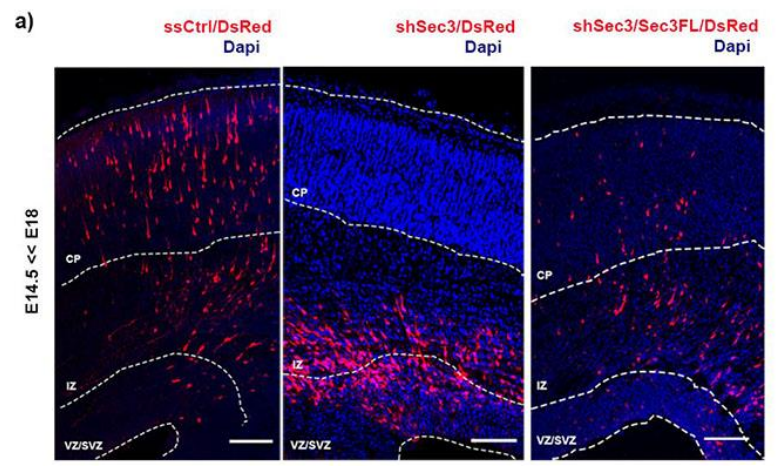

b)

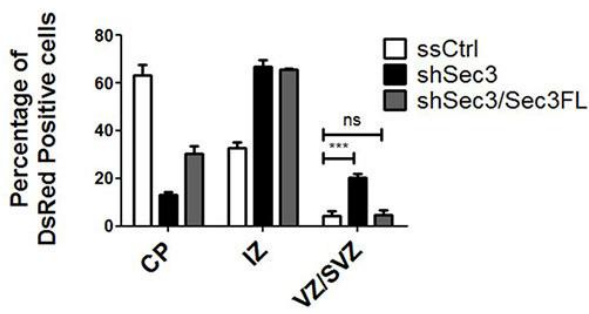

Fig 5-Sec3 is necessary for the regulation of cortical neuron migration. a) Representative images of brains co-electroporated at E15 and analyzed at E18 with control ssCtrl (first row), Sec3-targeted shRNA (second row) and Sec3-targeted shRNA/Sec3FL and in all cases with DsRed. Few DsRed positive cells were located in the cortical plate (CP) and the marginal zone (MZ) when Sec3-targeted shRNA expression was knocked down compared to control. Calibration bar $=10 \mu \mathrm{m}$. b) Quantification of the distribution of DsRed positive cells in Cortical Plate (CP), intermediate zone (IZ) and ventricular/subventricular zones (VZ/SVZ) as indicated in a) transfected with Sec3-targeted shRNA, Sec3-targeted shRNA/Sec3FL control sequence at E18. Bars represent mean \pm SEM of the percentage. A Two-way ANOVA Test with Bonferroni post-test was performed. $* * * \mathrm{P} \leqslant 0.001, * * \mathrm{P} \leqslant 0.01$, ns: non-significant, $\mathrm{n}=3$ independent experiments. At least 30 slices of brain were scored for each condition. 
a)

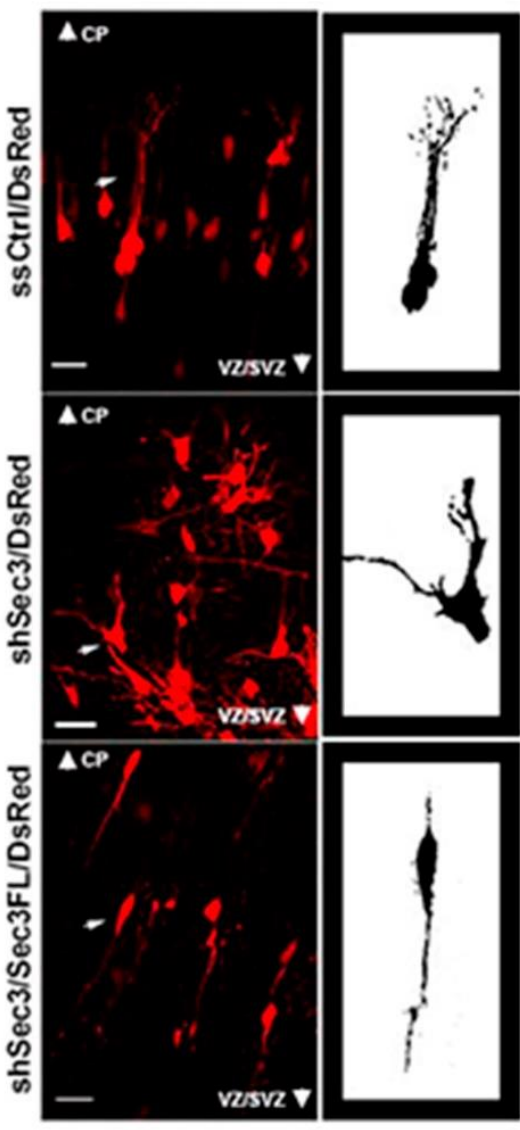

b)

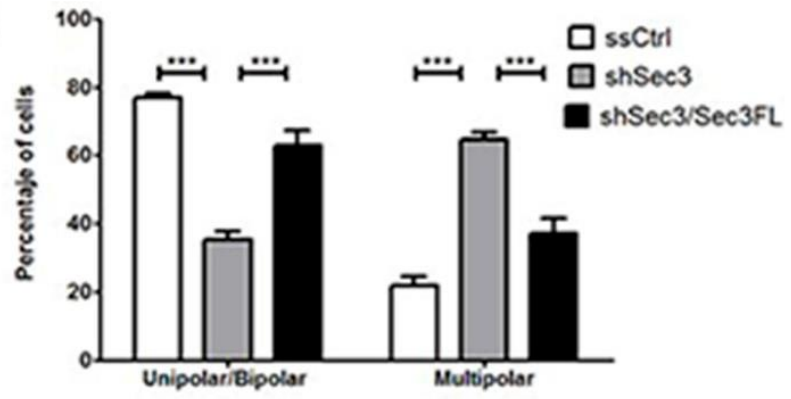

Fig 6-Sec3 is necessary for the polarity switch in cortical neurons. a) Representative images of brains co-electroporated with control ssCtrl (top), Sec3-targeted shRNA (middle) and Sec3-targeted shRNA/Sec3FL (bottom), in all cases with DsRed at E15 and analyzed at E18. DsRed positive cells with Sec3-targeted shRNA located in the intermediate zone showed predominantly a multipolar morphology in contrast to the ssCtrl or Sec3-targeted shRNA/Sec3FL cells that exhibit bipolar morphology. Calibration bar $=50 \mu \mathrm{m}$. b) Quantification of the morphology of DsRed positive cells as multipolar or Unipolar/bipolar in the intermediate zone (IZ). Bars represent mean \pm SEM of the percentage. A Two-way ANOVA Test with Bonferroni post-test was performed ***P $\leqslant 0.001 \mathrm{n}=3$ independent experiments. At least 30 images were scored for each condition. 


\section{Figures}

a)
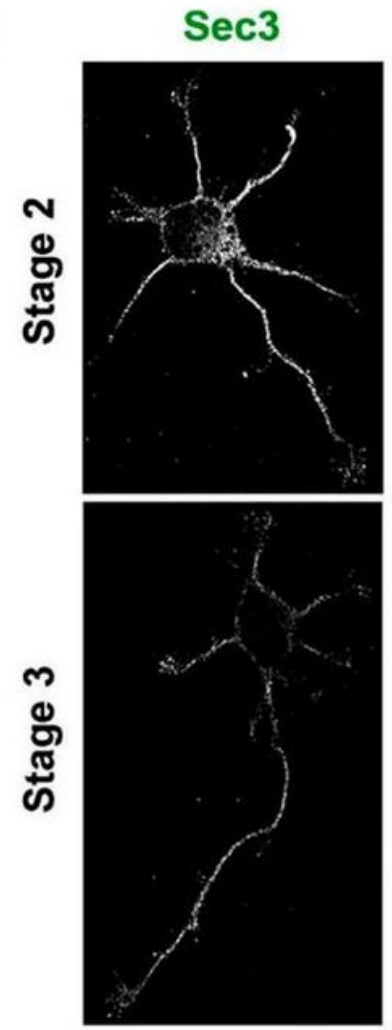

Tubulin
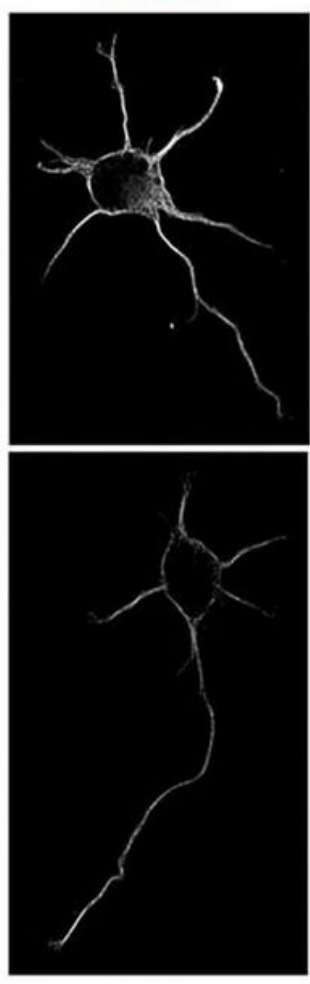

Merge
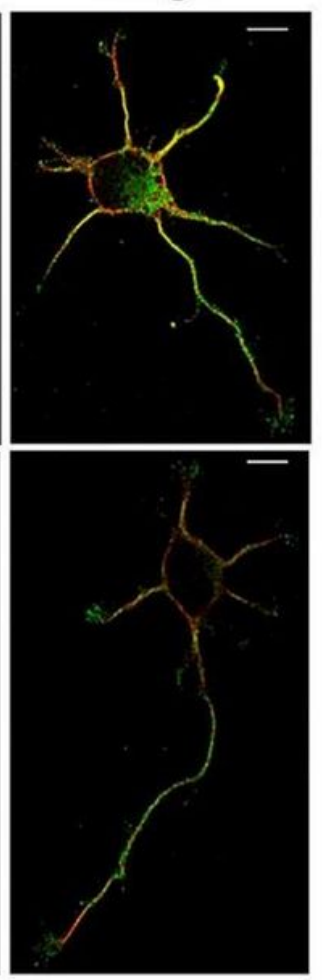

b)

12243672

$\operatorname{Sec} 3--==-102 \mathrm{kDa}$

Tub

$-55 k D a$

C)

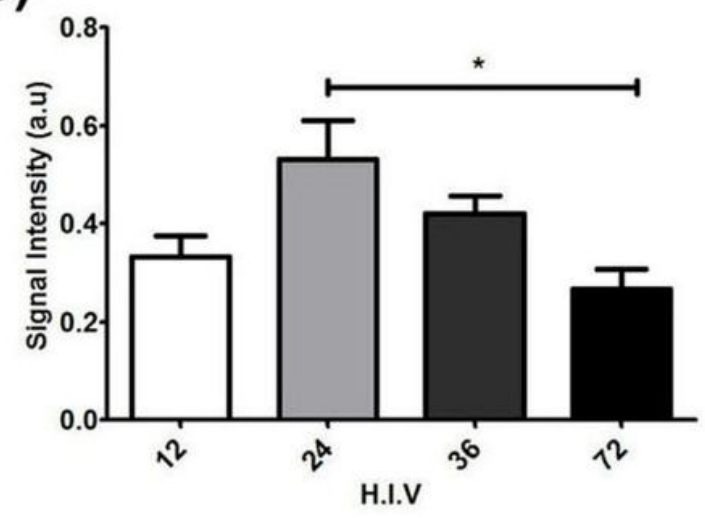

\section{Figure 1}

Sec3 is expressed previous to the polarization event, during the polarization and presents a significant diminution when the cells are fully polarized a) Representatives images of double immunofluorescence showing the distribution of Sec3 (green) and tyrosinated tubulin (red) in hippocampal pyramidal neurons in culture at late stage 2 after 18 (top) or stage 3 after 30 (bottom) H.I.V. Calibration bar $=20 \mu \mathrm{m}$. b) Western Blot of hippocampal cell lysates harvested at 12, 24, 36, or 72 H.I.V showing the expression of Sec3 (first row; apparent molecular weight $103 \mathrm{kDa}$ ) and tubulin (second row) as a loading control and secondary antibodies suitable for near infrared fluorescence. The obtained images were converted to grey scale and inverted. The results of one of three independent experiments are shown. The blots belong to the same experiment but were cropped for a better understanding. c) Quantification of Western blot for Sec3. Bars represent mean \pm SEM of the density measured in each band relative to tubulin density. A twoway ANOVA Test with Bonferroni post-test was performed. ${ }^{*} \otimes 0.05 n=3$ independent experiments. 
a)

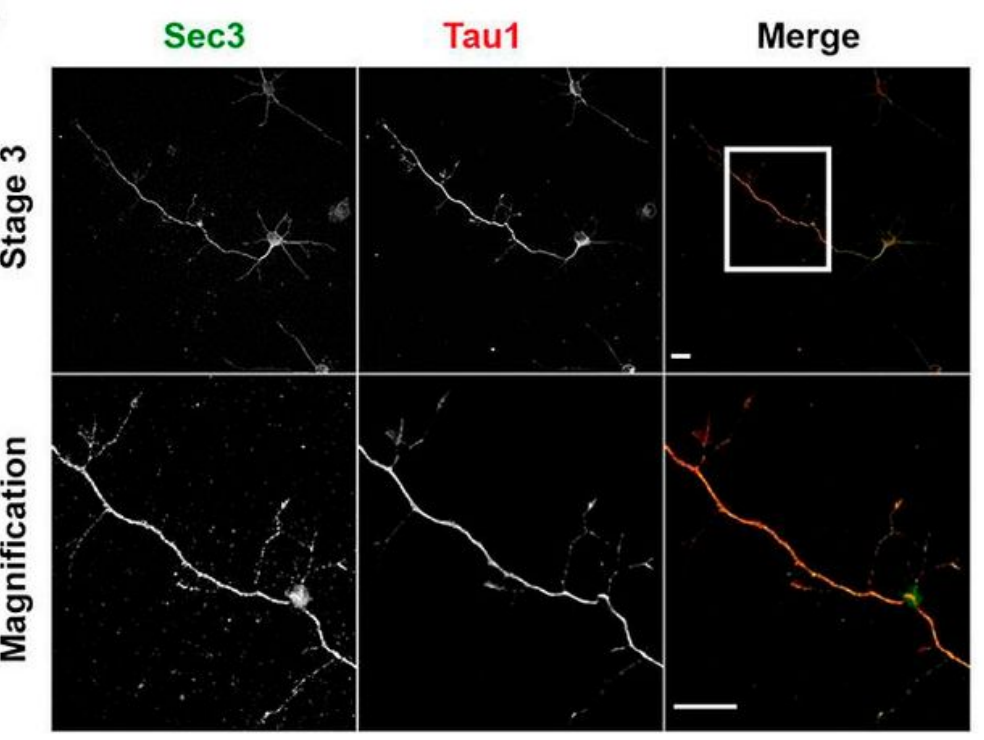

Sec3

Tubulin

d)

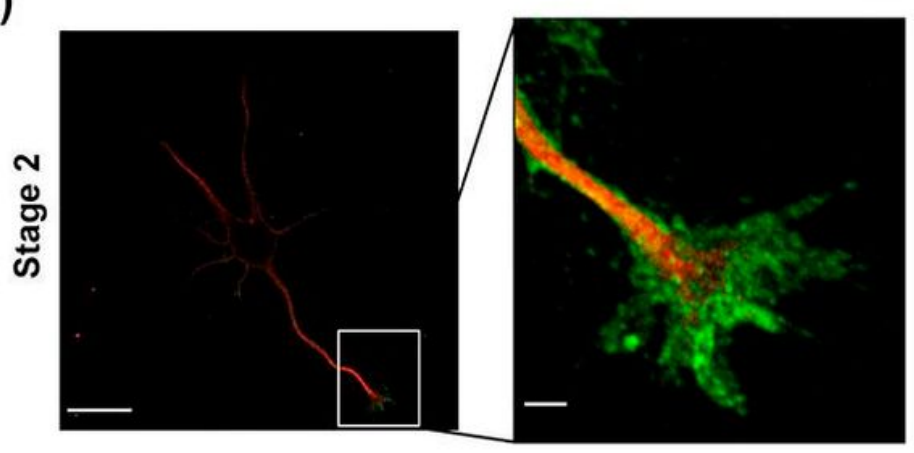

b)
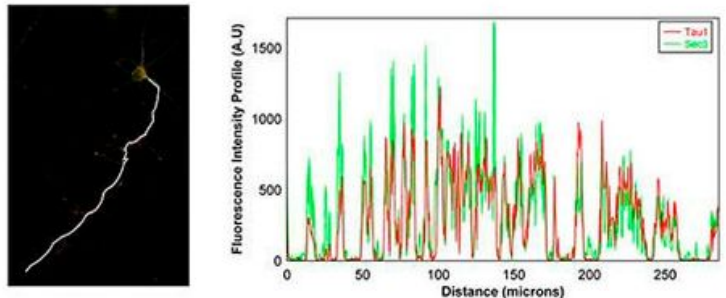

c)

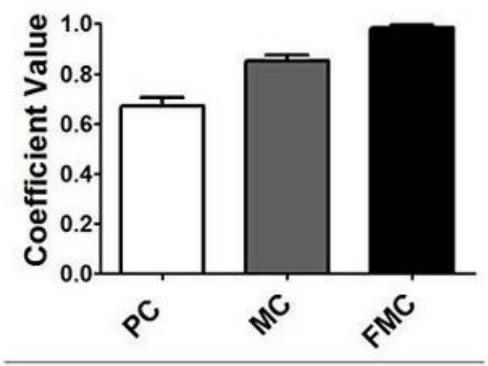

e)

$\begin{array}{rrrr} & \mathrm{Hm} & \text { GCPs } & \\ \text { Sec3 } & - & -102 \mathrm{kDa} \\ \text { Tub } & & -55 \mathrm{kDa}\end{array}$

Figure 2

Sec3 is localized at the axon and the growth cone. a) Representative images of double immunofluorescence micrographs showing the localization of Sec3 (green) or Tau1 (red) in hippocampal pyramidal neurons in culture at stage 3 after 24 H.I.V (top). A magnification of the axon is shown (bottom). Note the enriched co-localization at the distal axon. Calibration bar $=20 \mu \mathrm{m}$. b) Histogram (right) showing fluorescence intensity spatial profiles through the white line showing Sec3 (green channel) or and Tau1 (red channel) along white line showed in the merged image (left). c) Quantitative analysis of co-expression levels by Pearson's colocalization coefficient, Manders colocalization coefficient and Fractionary Mander's (M1 coefficient is expressed indicating Sec3 that colocalizes with Tau1 using JACoP, Image J. Bars represent mean \pm SEM of the coefficient. $n=3$ independent experiments 10 cells were scored for each coefficient measure. d) Representative images of double immunofluorescence micrographs showing the localization of Sec3 (green) or Tyr Tubulin (red) in axonal growth cone in hippocampal pyramidal neurons in culture at late stage 2 after 18 H.I.V. A magnification of the axon is shown (left). Calibration bar $=20 \mu \mathrm{m}$. e) Western blot of total 18-day rat embryo brain homogenate (first column) and GCPs (second column) showing the expression of Sec3 (first row; apparent molecular weight $103 \mathrm{kDa}$ ) and tubulin (second row) as a loading control and secondary 
antibodies suitable for near infrared fluorescence. The obtained images were converted to grey scale and inverted. The results of one of three independent experiments are shown. The blots belong to the same experiment but were cropped for a better understanding.

a)
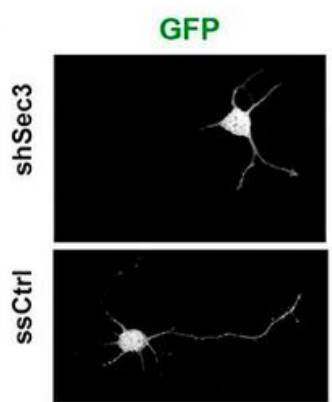

b)

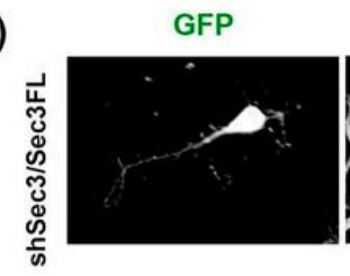

e)

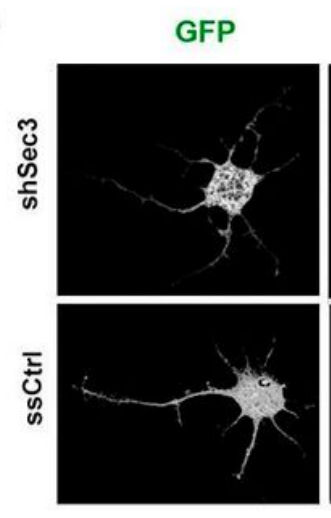

Tau1
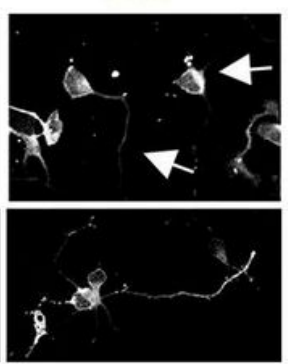

Tau1

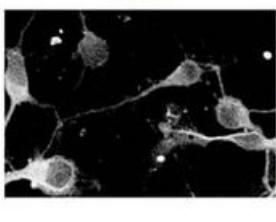

plGF1

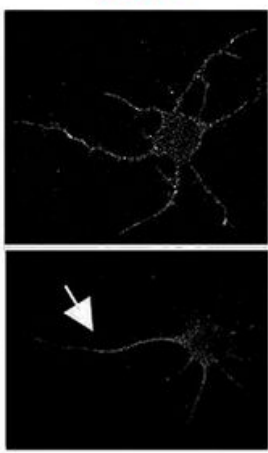

Sec3

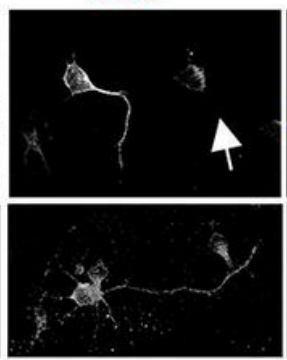

Merge

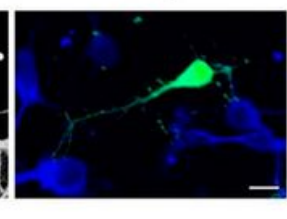

Tyr Tub

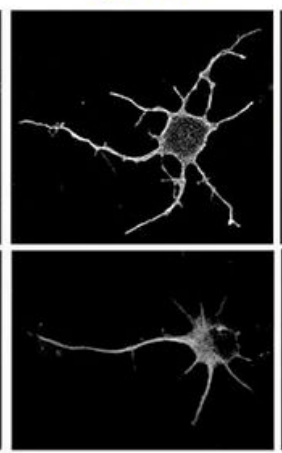

Merge

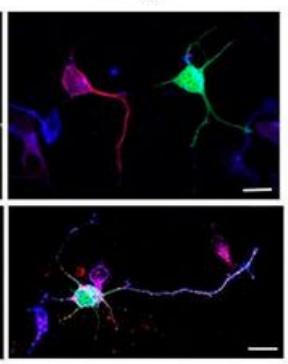

d)

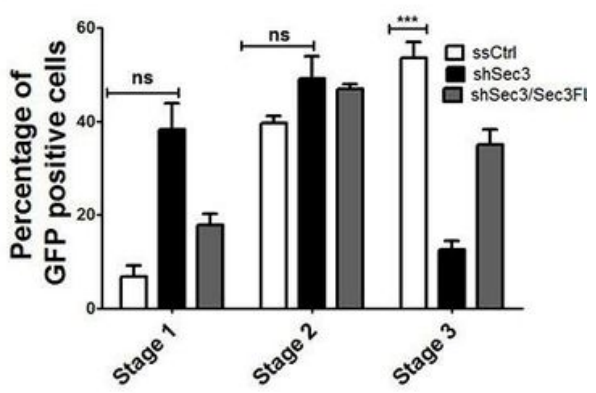

f)

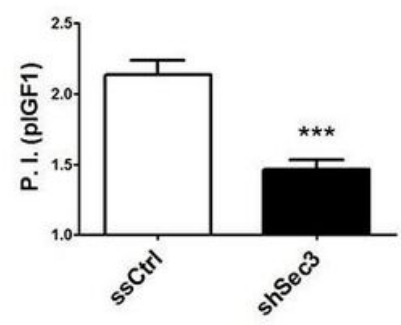

\section{Figure 3}

Sec3 is necessary for the establishment of neuronal polarity in mouse hippocampal neurons. a) Representative images of double immunofluorescence micrographs showing the localization of Sec3 (red, third column) or Tau-1 (blue, second column) and GFP as a transfection marker (first column) in hippocampal pyramidal neurons in culture at stage 3 after 24 H.I.V transfected with Sec3-targeted shRNA (shSec3, first row) and a scrambled RNA sequence (ssCtrl, second row). Note that the neurons transfected with Sec3-targeted shRNA (arrow) did not develop axons and did not target Tau-1 to any particular neurite. Note that cells transfected with an ssCtrl exhibit an axon-like process enriched in Tau-1. Calibration bar $=20 \mu \mathrm{m}$. b) Representative images of double immunofluorescence micrographs showing the localization of Tau-1 (blue, second column) and GFP as a transfection marker (first column) in hippocampal pyramidal neurons in culture at stage 3 after 24 H.I.V transfected with Sec3-targeted shRNA and Sec3FL. Note that the neurons transfected with Sec3-targeted shRNA and Sec3FL develop axons. Calibration bar $=20 \mu \mathrm{m}$. c) Western blots of mouse Neuro-2a harvested at $24 \mathrm{H}$.I.V showing protein levels 
in culture in the presence of different Sec3-targeted shRNA (first row; apparent molecular weight $103 \mathrm{kDa}$ ) and tubulin (second row) as a loading control and secondary antibodies suitable for near infrared fluorescence. The obtained images were converted to grey scale and inverted. The blots belong to the same experiment but were cropped for a better understanding. d) Quantification of neuronal stages of cells transfected with Sec3-targeted shRNA, Sec3-targeted shRNA with a full length Sec3 protein or control sequence after 24 H.I.V. Bars represent mean \pm SEM of the percentage. A Two-way ANOVA Test with Bonferroni post-test was performed. ${ }^{\star \star *} \mathrm{P} \otimes 0.001$, ns: non significance. $\mathrm{n}=3$ independent experiments. At least 100 neurons were scored for each condition. e) Representative images of double immunofluorescence micrographs showing the distributions of phosphorylated IGF-1r (red, second column), tyrosinated tubulin (blue, third column) and the transfection marker GFP (first column) in hippocampal pyramidal neurons in culture at stage 2 after $20 \mathrm{H}$.I.V transfected with Sec3-targeted shRNA (shSec3, first row) or a scrambled RNA sequence (ssCtrl, second row). Neurons were deprived of growth factors for $4 \mathrm{~h}$ and stimulated with $20 \mathrm{nM}$ IGF-1 for 2 min before fixation. Note the polarization of active (membrane-inserted) IGF-1 $r$ to one of the minor neurites of the cell transfected with ssRNA (bottom, arrow). In contrast, neurons transfected with Sec3 (bottom) failed to polarize the active IGF-1 $r$ to any neurite. Calibration bar $=20 \mu \mathrm{m}$. f) Quantification of a polarization index of active IGF-1r (P.I. IGF-1r.) calculated as the fluorescence intensity (A.U.) of the brightest minor neurite/average fluorescence intensity (A.U.) of the other minor neurites of the same cell. Bars represent mean \pm SEM of the P.I. Neurons were processed as in c. The polarization index is significantly higher in neurons transfected with scrambled sequence compared to those transfected with shSec3. A t-Test was performed ${ }^{\star \star *} \mathrm{P} \leq 0.001$. ns: non-significant $n=3$ independent experiments 10 cells were scored for each condition. 


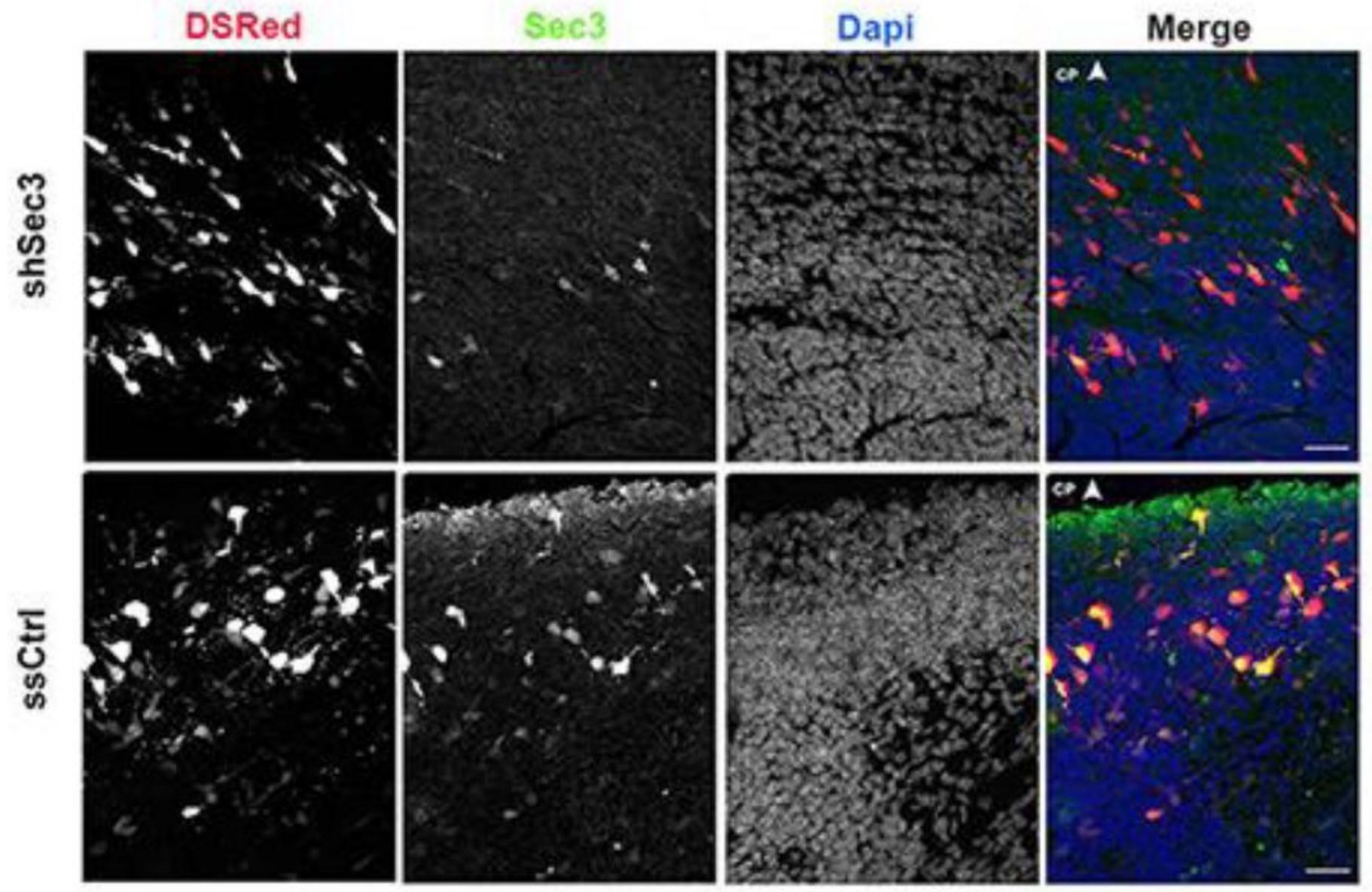

Figure 4

Electroporation with shSec3 reduces expression Sec3. a) Representative images of brains coelectroporated at E15 and analyzed at E17 with Sec3-targeted shRNA (top) or control ssCtrl (bottom) with DsRed showing the expression of DsRed positive cells (first row), Sec3 (green, second row) and Dapi (third row). The Cortical Plate (CP) is labeled for orientation.

a)

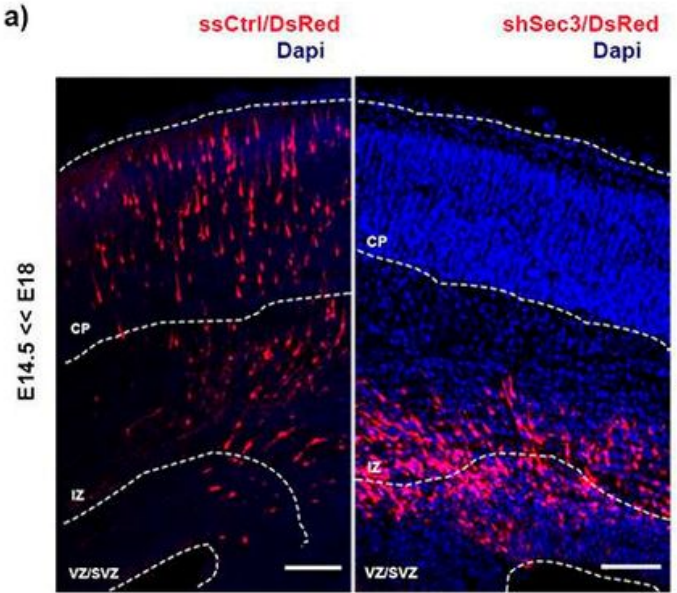

shSec3/Sec3FL/DsRed Dapi

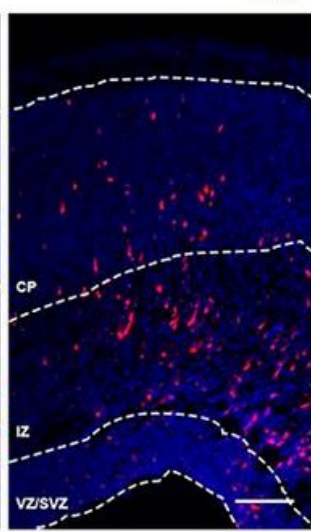

b)

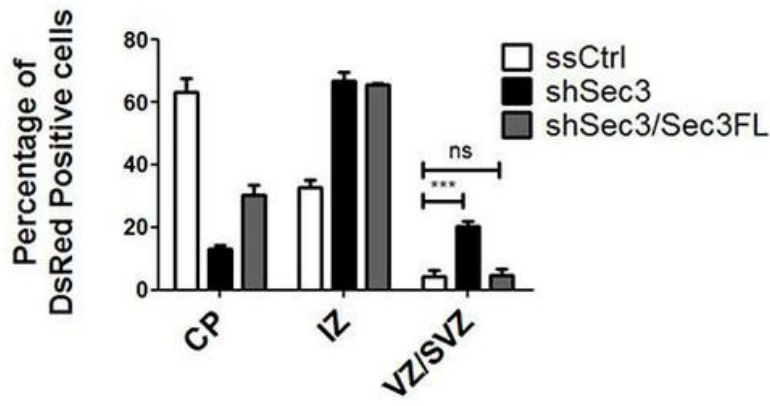

Figure 5 
Sec3 is necessary for the regulation of cortical neuron migration. a) Representative images of brains coelectroporated at E15 and analyzed at E18 with control ssCtrl (first row), Sec3-targeted shRNA (second row) and Sec3-targeted shRNA/Sec3FL and in all cases with DsRed. Few DsRed positive cells were located in the cortical plate (CP) and the marginal zone (MZ) when Sec3-targeted shRNA expression was knocked down compared to control. Calibration bar $=10 \mu \mathrm{m}$. b) Quantification of the distribution of DsRed positive cells in Cortical Plate (CP), intermediate zone (IZ) and ventricular/subventricular zones (VZ/SVZ) as indicated in a) transfected with Sec3-targeted shRNA, Sec3-targeted shRNA/Sec3FL control sequence at E18. Bars represent mean \pm SEM of the percentage. A Two-way ANOVA Test with Bonferroni post-test was performed. ${ }^{\star \star \star} \mathrm{P} \otimes 0.001,{ }^{\star \star} \mathrm{P} \otimes 0.01$, ns: non-significant, $\mathrm{n}=3$ independent experiments. At least 30 slices of brain were scored for each condition.

a)

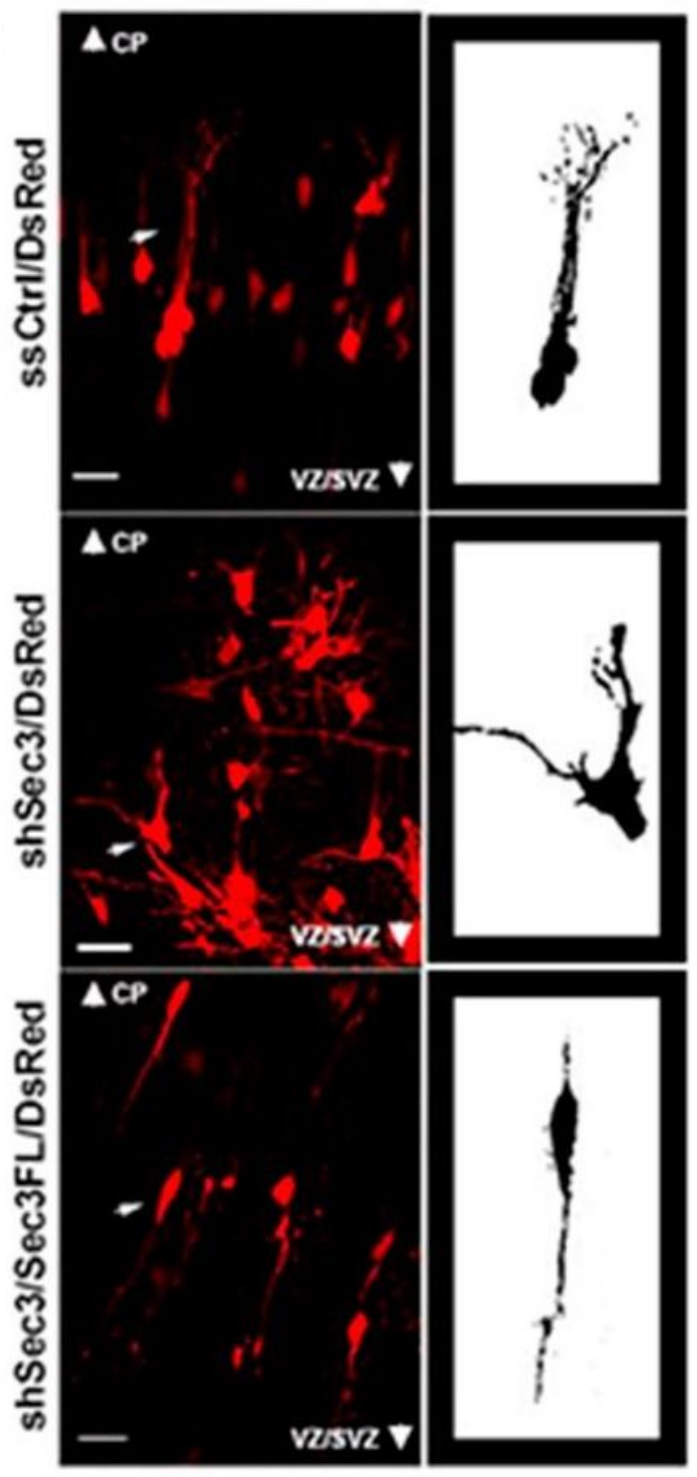

b)

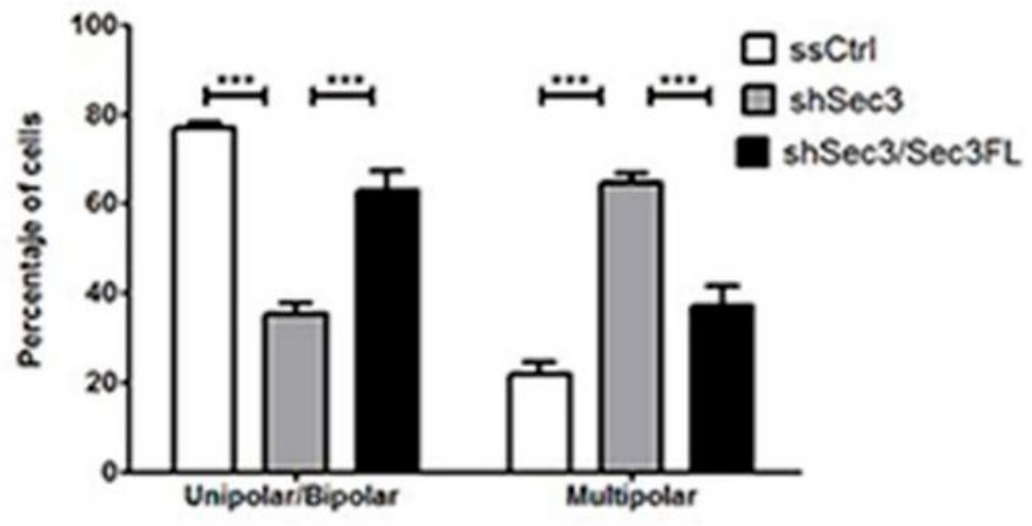

Figure 6

Sec3 is necessary for the polarity switch in cortical neurons. a) Representative images of brains coelectroporated with control ssCtrl (top), Sec3-targeted shRNA (middle) and Sec3-targeted shRNA/Sec3FL (bottom), in all cases with DsRed at E15 and analyzed at E18. DsRed positive cells with Sec3-targeted 
shRNA located in the intermediate zone showed predominantly a multipolar morphology in contrast to the ssCtrl or Sec3-targeted shRNA/Sec3FL cells that exhibit bipolar morphology. Calibration bar $=50 \mu \mathrm{m}$. b) Quantification of the morphology of DsRed positive cells as multipolar or Unipolar/bipolar in the intermediate zone (IZ). Bars represent mean \pm SEM of the percentage. A Two-way ANOVA Test with Bonferroni post-test was performed ${ }^{* *} \mathrm{P} \otimes 0.001 \mathrm{n}=3$ independent experiments. At least 30 images were scored for each condition.

\section{Supplementary Files}

This is a list of supplementary files associated with this preprint. Click to download.

- Supplementarymaterial.pdf 